\title{
TITLE:
}

\section{Consequentialism and Dynamic Consistency in Updating Ambiguous Beliefs}

AUTHOR(S):

Asano, Takao; Kojima, Hiroyuki

CITATION:

Asano, Takao ...[et al]. Consequentialism and Dynamic Consistency in Updating Ambiguous Beliefs. KIER Discussion Paper 2018, 987: 1-33

ISSUE DATE:

2018-03-14

URL:

http://hdl.handle.net/2433/236138

RIGHT: 


\title{
KIER DISCUSSION PAPER SERIES
}

\author{
KYOTO INSTITUTE \\ OF \\ ECONOMIC RESEARCH
}

\author{
Discussion Paper No.987 \\ "Consequentialism and Dynamic Consistency in \\ Updating Ambiguous Beliefs"
}

Takao Asano Hiroyuki Kojima

March 2018

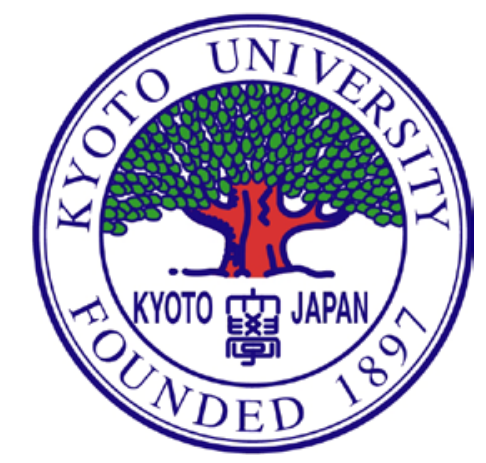

KYOTO UNIVERSITY

KYOTO, JAPAN 


\title{
Consequentialism and Dynamic Consistency in Updating Ambiguous Beliefs*
}

\author{
Takao Asano ${ }^{\dagger}$ Hiroyuki Kojima ${ }^{\ddagger}$
}

This Version: March 14, 2018

\begin{abstract}
By proposing the notions of upper-constrained dynamic consistency and lower-constrained dynamic consistency that are weaker axioms than dynamic consistency, this paper axiomatizes the Dempster-Shafer updating rule and naive Bayes' updating rule within the framework of Choquet expected utility. Based on the notion of conditional comonotonicity, this paper also provides an axiomatization of consequentialism under Choquet expected utility. Furthermore, based on the idea of the mean preserving rule, this paper provides a unified approach for distinguishing capacity updating rules (the DempsterShafer updating rule, naive Bayes' updating rule, and Fagin-Halpern updating rule) according to the degree of dynamic consistency.
\end{abstract}

JEL Classification Numbers: C71, D81, D90

Key Words: Dynamic Consistency, Consequentialism, Choquet Expected

Utility, Conditional Comonotonicity, Conditional Preferences,

Dempster-Shafer Updating Rule, Naive Bayes' Updating Rule,

Fagin-Halpern Updating Rule

${ }^{*}$ We acknowledge an anonymous reviewer and the co-editor, Mark Machina, whose comments improve this paper substantially. We are grateful to Youichiro Higashi, Hidetoshi Komiya, Hiroyuki Ozaki, Shin'ichi Suda, Masayuki Yao, and participants at Nagoya University, Keio University, and China Meeting of Econometric Society 2016 (Chengdu, China). This research is financially supported by the JSPS KAKENHI Grant Numbers 17K03806, 16K03558, 26380240, 25380239, and 23000001, and the Joint Research Program of KIER.

${ }^{\dagger}$ Faculty of Economics, Okayama University, 3-1-1, Tsushimanaka, Kita-ku, Okayama 700-8530, Japan. e-mail: asano@e.okayama-u.ac.jp. Tel: +81-86-251-7558

$\ddagger$ Department of Economics, Teikyo University, 359, Ohtsuka, Hachioji, Tokyo 192-0395, Japan. e-mail: hkojima@main.teikyo-u.ac.jp 


\section{Introduction}

It is difficult to precisely forecast what will happen in the future since we have insufficient information about the state of the world. Such "uncertainty" affects the behavior of a decision maker (DM). Further, obtaining new information affects a DM's behaviors in a dynamic choice environment. For example, investors faced with portfolio allocation problems must consider what will happen in the future, which will affect stock prices and the returns on their portfolios. Moreover, they will update their beliefs if they obtain some information that affects individual stock prices or stock markets. Under "uncertainty," how will a DM update her beliefs after obtaining new information and how will a DM's preferences be formulated? What properties should be taken into account in a dynamic choice environment? To analyze such situations, particularly in a dynamic environment, we should pay attention to at least two properties. Dynamic consistency and consequentialism play significant roles in the literature. However, as explained by Example 2 later in this section, these two properties cannot be retained simultaneously. The purpose of this paper is therefore to explore the extent to which dynamic consistency should be weakened with retaining consequentialism for axiomatizing three updating rules (the Dempster-Shafer updating rule, naive Bayes' updating rule, and Fagin-Halpern updating rule) under Knightian uncertainty or ambiguity, ${ }^{1}$ particularly within the framework of Choquet expected utility (CEU).

The importance of the distinction between risk and Knightian uncertainty or ambiguity has been recognized in the literature since Ellsberg (1961). While risk is situations described by known probabilities, ambiguity or Knightian uncertainty is situations where probabilities are neither given nor calculable. To overcome the shortcoming of subjective expected utility (SEU) (Savage (1954)) raised by Ellsberg (1961), several works have been proposed in the literature. ${ }^{2}$ We provide the following examples to explain how dynamic consistency and consequentialism are violated. To put it simply, dynamic consistency states that for any $\operatorname{acts}^{3} f$ and $g$ and for any event $A$, when a DM prefers $f$ to $g$ without observing $A$, and $f$ and $g$ coincide on $A^{c}$, she should prefer $f$ to $g$ after observing that $A$ occurred. Consequentialism means that for any event $A$, conditional preferences on $A$ only depend on the outcomes inside the observed event $A$, but are not affected by the outcomes on $A^{c}$.

\footnotetext{
${ }^{1}$ Throughout this paper, ambiguity and Knightian uncertainty are used interchangeably.

${ }^{2}$ For an axiomatization of CEU, see Schmeidler (1989). For an axiomatization of max-min expected utility (MEU), see Gilboa and Schmeidler (1989).

${ }^{3}$ For the definition of acts or lotteries, see subsection 2.1 .
} 
Example 1 (Ellsberg (1961)). Suppose that an urn contains 90 balls. The number of red balls is 30 and the number of black and yellow balls is 60 . Consider a bet (or a lottery) on drawing a ball from this urn. We get $\$ 100$ depending on the color. The DM has to choose between two bets $\left(f, f^{\prime}\right)$ and $\left(g, g^{\prime}\right)$ :

$$
\begin{gathered}
f=\left(\begin{array}{ccc}
100 & \text { if } & \omega \in R \\
0 & \text { if } & \omega \in B \\
0 & \text { if } & \omega \in Y
\end{array}\right) \quad f^{\prime}=\left(\begin{array}{ccc}
0 & \text { if } & \omega \in R \\
100 & \text { if } & \omega \in B \\
0 & \text { if } & \omega \in Y
\end{array}\right) \\
g=\left(\begin{array}{cll}
100 & \text { if } & \omega \in R \\
0 & \text { if } & \omega \in B \\
100 & \text { if } & \omega \in Y
\end{array}\right) \quad g^{\prime}=\left(\begin{array}{ccc}
0 & \text { if } & \omega \in R \\
100 & \text { if } & \omega \in B \\
100 & \text { if } & \omega \in Y
\end{array}\right)
\end{gathered}
$$

For example, bet $f^{\prime}$ states that we obtain $\$ 100$ if we draw a black ball and nothing otherwise. Many respondents prefer $f$ to $f^{\prime}$ and $g^{\prime}$ to $g$, denoted by $f \succ f^{\prime}$ and $g^{\prime} \succ g$, respectively. However, these choices cannot be explained within the framework of SEU, which is called Ellsberg's paradox.

In the following example, we consider a dynamic version of Ellsberg (1961), which is based on Dominiak and Lefort (2011).

Example 2. We consider two stages, the ex-ante stage and the interim stage. The ex-ante stage $(t=0)$ corresponds to Example 1 in which the DM has no information. Then, many respondents prefer $f$ to $f^{\prime}$ and $g^{\prime}$ to $g$, denoted by $f \succ f^{\prime}$ and $g^{\prime} \succ g$, respectively. In the interim stage $(t=1)$, one ball is drawn from the urn at random and the DM is informed that the ball is not yellow, i.e., $\{R, B\}$. We denote the conditional preferences by $\succ_{\{R, B\}}$. On the one hand, since $f \succ f^{\prime}$ and $\left.f\right|_{\{R, B\}^{c}}=\left.f^{\prime}\right|_{\{R, B\}^{c}}$, dynamic consistency implies $f \succ_{\{R, B\}} f^{\prime}$. Similarly, since $g^{\prime} \succ g$ and $\left.g^{\prime}\right|_{\{R, B\}^{c}}=\left.g\right|_{\{R, B\}^{c}}$, dynamic consistency implies $g^{\prime} \succ_{\{R, B\}} g$. On the other hand, since $\left.f\right|_{\{R, B\}}=\left.g\right|_{\{R, B\}}$, consequentialism implies $f \sim_{\{R, B\}} g$. Similarly, since $\left.f^{\prime}\right|_{\{R, B\}}=\left.g^{\prime}\right|_{\{R, B\}}$, consequentialism implies $f^{\prime} \sim_{\{R, B\}} g^{\prime}$. Furthermore, consequentialism implies that if $f \succ_{\{R, B\}} f^{\prime}$, then $g \succ_{\{R, B\}} g^{\prime}$ and vice versa. Similarly, consequentialism implies that if $g^{\prime} \succ_{\{R, B\}} g$, then $f^{\prime} \succ_{\{R, B\}} f$ and vice versa. Therefore, if conditional preferences respect dynamic consistency, then consequentialism is violated. On the contrary, if conditional preferences respect consequentialism, then dynamic consistency is violated.

As pointed out in Dominiak and Lefort (2011), when we consider updating rules under ambiguity, we must relax either the property of dynamic consistency or the property of consequentialism. Dominiak et al. (2012) experimentally find that a 
significant majority of subjects violate dynamic consistency rather than consequentialism. However, as they state, whether one property is more plausible than another remains to be solved. At least, three approaches to this problem exist in the literature. The first approach is to retain both properties. Restricting some domains of acts and events, under MEU, Sarin and Wakker (1998) and Epstein and Schneider (2003) maintain these two properties. The second and third approaches are to drop one of the properties. While Gilboa and Schmeidler (1993), Pires (2002), Eichberger et al. (2007), and Siniscalchi (2011) maintain consequentialism and drop dynamic consistency, ${ }^{4}$ Machina (1989) and Hanany and Klibanoff (2007) maintain dynamic consistency and drop consequentialism. ${ }^{5}$ This paper proposes a fourth approach. That is, by retaining the property of consequentialism and some kinds of dynamic consistency that are weaker than dynamic consistency, we axiomatize the Dempster-Shafer updating rule and naive Bayes' updating rule under CEU. For this purpose, based on the notion of conditional comonotonicity, ${ }^{6}$ this paper also provides an axiomatization of consequentialism under CEU.

The effects of obtaining new information on individual decision making are worth investigating within the frameworks of CEU and MEU as well as SEU. Ghirardato (2002) provides a simple axiomatization for conditional preferences within the framework of the Savage acts in which a DM's beliefs are updated by the Bayesian updating rule. On the one hand, from the viewpoint of statistics, some updating rules have been proposed by Dempster (1967, 1968), Shafer (1976), and Fagin and Halpern (1991). On the other hand, from the viewpoint of decision theory, axiomatizations of conditional preferences within the framework of CEU have been proposed by Gilboa and Schmeidler (1993), Pires (2002), and Wang (2003). ${ }^{7}$ As mentioned in subsection 2.2, while a DM whose preferences are represented by SEU satisfies both dynamic consistency and consequentialism, updating rules in CEU do not necessarily satisfy dynamic consistency; in particular, the three updating rules for capacities (the Dempster-Shafer, naive Bayes', and Fagin-Halpern updating rules) do not sat-

\footnotetext{
${ }^{4}$ Gilboa and Schmeidler (1993), Pires (2002), and Eichberger et al. (2007) consider updating rules within the framework of CEU. Siniscalchi (2011) analyzes a general framework that does not restrict attention to any specific model, for example, CEU or MEU.

${ }^{5}$ Machina (1989) considers a broader class of non-expected utilities than Hanany and Klibanoff (2007) that analyze updating rules for MEU. Epstein and Le Breton (1993) show that under some assumptions, dynamic consistency for CEU implies that the DM's beliefs must be additive.

${ }^{6}$ The notion of conditional comonotonicity is an extension of comonotonicity. See Section 3.

${ }^{7}$ Wang (2003) considers a complicated framework and provides a set of axioms that endogenize information filtration. Epstein and Schneider (2003) propose a set of axioms of conditional preferences within the framework of MEU. Ghirardato et al. (2008) and Hanany and Klibanoff (2007, 2009) propose a set of axioms of conditional preferences under more general frameworks than MEU.
} 
isfy dynamic consistency, but satisfy consequentialism. On the one hand, Dominiak et al. (2012) experimentally find that a significant majority of subjects violate dynamic consistency rather than consequentialism. On the other hand, dynamic consistency plays an important role in intertemporal decision making within the framework of not only the standard expected utility theory but also non-expected utility theories. Machina (1989) points out the case in which some kind of inconsistency emerges if the DM in intertemporal choice situations does not satisfy dynamic consistency. Therefore, the following viewpoints are worth investigating. To what extent do updating rules for capacities with consequentialism satisfy dynamic consistency? What kinds of axioms are satisfied by updating rules with consequentialism within the framework of CEU? For the former viewpoint, by proposing the notions of upper-constrained dynamic consistency and lower-constrained dynamic consistency (see Section 4 for the definitions) that are weaker axioms than dynamic consistency, this paper axiomatizes the Dempster-Shafer and naive Bayes' updating rules under CEU. For the latter viewpoint, by proposing the notions of conditional comonotonicity and consequentialism-capacity (see subsection 2.2 for the definition), we provide an axiomatization of the consequentialism-capacities under CEU. Furthermore, we characterize the three updating rules for capacities based on consequentialism-capacities. In addition, based on the notion of the mean preserving rule (MPR) (see Section 5 for the definition), we characterize the Fagin-Halpern updating rule together with the Dempster-Shafer and naive Bayes' updating rules under CEU. As a related paper, based on consequentialism, Eichberger et al. (2007) axiomatize the Fagin-Halpern updating rule. In the spirit of their paper, this paper provides a unified approach for distinguishing capacity updating rules according to the degree of dynamic consistency. The notion of the MPR enables us to illuminate the properties of these three updating rules from a consistent viewpoint.

The organization of this paper is as follows. Section 2 provides the notions of the updating rules and the definitions of Choquet integrals. It also presents the notions of dynamic consistency and consequentialism. Section 3 provides an axiomatization of consequentialism-capacities. Section 4 provides the notions of upperconstrained dynamic consistency and lower-constrained dynamic consistency and characterizes the Dempster-Shafer updating rule and naive Bayes' updating rule by upper-constrained dynamic consistency and lower-constrained dynamic consistency, respectively. Section 5 characterizes not only these two updating rules but also the Fagin-Halpern updating rule based on the MPR. Section 6 concludes this paper. Some proofs are relegated to the Appendix. 


\section{Updating Rules, Consequentialism, and Dynamic Consistency}

In this section, we present the notions of the updating rules for probabilities and capacities and the definitions of Choquet integrals. Furthermore, we present the notions of dynamic consistency and consequentialism.

\subsection{Preliminaries and Updating Rules}

Let $\Omega$ be a finite state space whose generic element is denoted by $\omega$. A subset $E \subseteq \Omega$ is called an event. Let $2^{\Omega}$ be the power set of $\Omega$ and $\mathcal{F} \subseteq 2^{\Omega}$ be an algebra of $\Omega$. Let $(\Omega, \mathcal{F})$ be a measurable space. A function $p: \mathcal{F} \rightarrow[0,1]$ is called a probability if (i) $p(A \cup B)=p(A)+p(B)$ for all $A, B \in \mathcal{F}$ with $A \cap B=\emptyset$ and (ii) $p(\Omega)=1, p(\emptyset)=0$. A capacity is an extension of probabilities that requires only monotonicity instead of additivity (i). That is, a function $v: \mathcal{F} \rightarrow[0,1]$ is said to be a capacity if (iii) $v(A) \leq v(B)$ for all $A, B \in \mathcal{F}$ with $A \subseteq B$, and (iv) $v(\Omega)=1, v(\emptyset)=0$. Let $X$ be the non-empty set of all deterministic outcomes and $Y$ be the set of all distributions over $X$ with finite supports, that is, $Y=\{y: X \rightarrow$ $[0,1] \mid y(x) \neq 0$ for finitely many $x \in X$ and $\left.\sum_{x \in X} y(x)=1\right\}$, which is a mixture space. ${ }^{8}$ Following Anscombe and Aumann (1963), define a lottery act or act as a $Y$ valued function on $\Omega$ whose range is a finite subset of $Y$. For notational simplicity, we identify $x \in X$ with the Dirac measure $\delta_{x} \in Y$, i.e., $\delta_{x}$ is the probability measure that assigns probability one to $\{x\}$. The set of all functions from $\Omega$ to $Y$ is denoted by $L_{0}$. The set of all constant functions in $L_{0}$ is denoted by $L_{c}$, and the elements of $L_{c}$ are called constant acts. For all $f, g \in L_{0}$ and $\alpha \in[0,1]$, the compound lottery is defined by $(\alpha f+(1-\alpha) g)(\omega) \equiv \alpha f(\omega)+(1-\alpha) g(\omega)$ for all $\omega \in \Omega$. We assume that the DM has a binary relation $\succeq$ on $L_{0}$, which is called an unconditional preference, and the conditional preferences of the DM who observes an event $A$ are denoted by a binary relation $\succeq_{A}$ on $L_{0}$ for each $A \in \mathcal{F}$. Throughout this paper, we identify $\succeq_{\Omega}$ with $\succeq$. We denote by $\left(g, A ; f, A^{c}\right)$ the function that coincides with $g$ on $A$ and with $f$ on $A^{c}$. Furthermore, let $\left.f\right|_{A}$ denote the restriction of an act $f$ to a set $A$. Let $u: Y \rightarrow \mathbb{R}$ be a utility function and $f: \Omega \rightarrow Y$ be an act. SEU states that under a set of axioms, the DM's preference $\succeq$ is represented by a probability measure $p$ and a utility function $u$, that is, $f \succeq g \Leftrightarrow \int_{\Omega} u(f) d p \geq \int_{\Omega} u(g) d p$, where the integrals are in the sense of the Lebesgue integrals. Let $v(T)$ be the number which DM assigns

\footnotetext{
${ }^{8}$ Let $Y$ be the set of all distributions over $X$ with finite supports. Define a mixing operation as follows. For all $y, y^{\prime} \in Y$ and all $\alpha \in[0,1], \alpha y+(1-\alpha) y^{\prime} \in Y$ is given by $\left(\alpha y+(1-\alpha) y^{\prime}\right)(x)=$ $\alpha y(x)+(1-\alpha) y^{\prime}(x)$. Then, the set $Y$ with this mixing operation is a mixture space. For example, see Gilboa (2009).
} 
to an event $T$. Example 1 in the Introduction implies that

$$
v(R)>v(B) \text { and } v(R \cup Y)<v(B \cup Y) .
$$

If $v$ is additive (i.e., a probability measure), these inequalities do not hold simultaneously. By replacing the additivity with the non-additivity, Schmeidler (1989) intends to explain Ellsberg's paradox. Schmeidler (1989) proposes CEU, which states that under a set of axioms, the DM's preference $\succeq$ is represented by a capacity $v$ and a utility function $u$, that is, $f \succeq g \Leftrightarrow \int_{\Omega} u(f) d v \geq \int_{\Omega} u(g) d v$, where the integrals are in the sense of the Choquet integrals. The definition of Choquet integrals is as follows. Let $\mathbb{R}^{\Omega}=\{x \mid x: \Omega \rightarrow \mathbb{R}\}$ be the set of all real-valued functions on $\Omega$. Let $1_{E}$ be the indicator function of a set $E \in 2^{\Omega}$. For $x \in \mathbb{R}^{\Omega}$ and a capacity $v$, the Choquet integral of $x$ with respect to $v$ is defined as $\int_{\Omega} x d v=\int_{0}^{\infty} v(x \geq \alpha) d \alpha+\int_{-\infty}^{0}(v(x \geq \alpha)-1) d \alpha$, where $v(x \geq \alpha)=v(\{\omega \in \Omega \mid x(\omega) \geq \alpha\})$. Let $E_{1}, E_{2}, \ldots, E_{n}$ be a partition of $\Omega$ and $f=\sum_{i=1}^{n} x_{i} 1_{E_{i}}$ with $x_{1} \geq x_{2} \geq \cdots \geq x_{n}$ be a step function. Then, the Choquet integral can be written as follows:

$$
\int_{\Omega} f d v=\sum_{j=1}^{n-1}\left(x_{j}-x_{j+1}\right) v\left(\cup_{i=1}^{j} E_{i}\right)+x_{n} .
$$

Under CEU, it follows from Inequality (1) that $\int_{\Omega} u(f) d v=(u(100)-u(0)) v(R)+$ $u(0)>(u(100)-u(0)) v(B)+u(0)=\int_{\Omega} u\left(f^{\prime}\right) d v$ and $\int_{\Omega} u(g) d v=(u(100)-u(0)) v(R \cup$ $Y)+u(0)<(u(100)-u(0)) v(B \cup Y)+u(0)=\int_{\Omega} u\left(g^{\prime}\right) d v$, which resolves Ellsberg's paradox. Contrary to the case of SEU, these two inequalities hold simultaneously due to $v$ 's non-additivity.

To investigate the effects of obtaining new information on individual decision making, we consider the following updating rules. In probability theory, it is common to consider the Bayes' rule as the unique updating rule. Let $p$ be a probability on $(\Omega, \mathcal{F})$, and let $p(A)>0$ for $A \in \mathcal{F}$. Observing such an event $A$, the Bayes' rule (the conditional probability) is defined by

$$
p_{A}(S)=\frac{p(A \cap S)}{p(A)} .
$$

In contrast to probability theory, the theory of capacities proposes various updating rules. Among them, the Dempster-Shafer updating rule (Dempster (1968), Shafer (1976)), naive Bayes' updating rule (Gilboa and Schmeidler (1993)), and Fagin-Halpern updating rule or generalized Bayesian updating rule (Dempster (1967), Fagin and Halpern (1991)) are well known. Let $A \in \mathcal{F} \backslash\{\Omega, \emptyset\}$ be an event. Let $v$ 
be a capacity on $(\Omega, \mathcal{F})$. The Dempster-Shafer updating rule is defined by

$$
v_{A}^{D S}(S)=\frac{v\left(S \cup A^{c}\right)-v\left(A^{c}\right)}{1-v\left(A^{c}\right)},
$$

the naive Bayes' updating rule is defined by

$$
v_{A}^{N B}(S)=\frac{v(S \cap A)}{v(A)},
$$

and the Fagin-Halpern updating rule is defined by

$$
v_{A}^{F H}(S)=\frac{v(S \cap A)}{v(S \cap A)+1-v\left(S \cup A^{c}\right)},
$$

where all the denominators are assumed to be non-zero.

Throughout this paper, for ease of notation, we denote the three updating rules for capacities, $v_{A}^{D S}, v_{A}^{N B}$, and $v_{A}^{F H}$ by $v_{A}^{*}$ for a given event $A \in \mathcal{F}$, where $*$ corresponds to the Dempster-Shafer updating rule, naive Bayes' updating rule, and Fagin-Halpern updating rule. In subsection 2.2, we mention the rationale for these three updating rules in detail. The following two lemmas hold for the three updating rules.

Lemma 1. Let $v_{A}^{*}$ be the three updating rules for a capacity $v$. If $v$ is additive, then for any $S \in \mathcal{F}, v_{A}^{*}(S)$ coincides with $v(A \cap S) / v(A)$.

This lemma states that if a capacity $v$ is additive, then the three updating rules for $v$ are equal to the Bayes' rule for a probability measure $p$.

Lemma 2. Let $v$ be a capacity and $v_{A}^{*}$ be the three updating rules for $v$. Then, for any $S \in \mathcal{F}$,

$$
v_{A}^{*}(A \cap S)=v_{A}^{*}(S)
$$

\subsection{Consequentialism and Dynamic Consistency}

In this subsection, we investigate whether CEU satisfies consequentialism and dynamic consistency.

Definition 1. Let $A \in \mathcal{F}$ be an event, $v_{A}$ be a capacity, and $u: X \rightarrow \mathbb{R}$ be a utility function. An unconditional or conditional preferences, $\succeq_{A}$, are said to be captured by a capacity $v_{A}$ and a utility function $u$ if $f \succeq_{A} g \Leftrightarrow \int_{\Omega} u(f) d v_{A} \geq \int_{\Omega} u(g) d v_{A}$.

For simplicity, in the following, we say that a conditional preference $\succeq_{A}$ is captured by a capacity $v_{A}$. To consider the updating rules under SEU and CEU, we should analyze consequentialism and dynamic consistency. The following definition is based on Hammond $(1988,1989)$. 
Definition 2 (Consequentialism). Let $A \in \mathcal{F} \backslash\{\Omega, \emptyset\}$ be an event. A conditional preference $\succeq_{A}$ is said to satisfy consequentialism if the following condition holds: For any $f, g \in L_{0}$, if $\left.f\right|_{A}=\left.g\right|_{A}$, then $f \sim_{A} g$.

Consequentialism means that the conditional preferences on $A$ only depend on the outcomes inside the observed event $A$, but are not affected by the outcomes on $A^{c}$. Next, we propose the notion of consequentialism-capacity (C-capacity), which plays a significant role in this paper.

Definition 3 (Consequentialism-Capacity). Let $A \in \mathcal{F} \backslash\{\Omega, \emptyset\}$ be an event. Suppose that a conditional preference $\succeq_{A}$ is captured by a capacity $v_{A}$. Such a capacity $v_{A}$ is said to be a consequentialism-capacity (C-capacity) if $\succeq_{A}$ satisfies consequentialism.

Now, the following proposition provides a necessary and sufficient condition for C-capacities.

Proposition 1. Let $A \in \mathcal{F} \backslash\{\Omega, \emptyset\}$ be an event. Suppose that a conditional preference $\succeq_{A}$ is captured by a capacity $v_{A}$. Then, $v_{A}$ is a C-capacity if and only if $v_{A}(S)=v_{A}(S \cap A)$ for all $S \in \mathcal{F}$.

Proof. See the Appendix.

The following corollary immediately follows from Lemma 2 and Proposition 1.

Corollary 1. Let $A \in \mathcal{F} \backslash\{\Omega, \emptyset\}$ be an event. Then, $v_{A}^{D S}, v_{A}^{N B}$, and $v_{A}^{F H}$ are all C-capacities.

Next, the definition of dynamic consistency is in order.

Definition 4 (Dynamic Consistency). Let $A \in \mathcal{F} \backslash\{\Omega, \emptyset\}$ be an event. A conditional preference $\succeq_{A}$ is said to satisfy dynamic consistency if the following condition holds: For any $f, g \in L_{0}$, if $f \succeq g$ and $\left.f\right|_{A^{c}}=\left.g\right|_{A^{c}}$, then $f \succeq_{A} g$.

Dynamic consistency means that for any acts $f$ and $g$, when the DM prefers $f$ to $g$ without observing $A$, and $f$ and $g$ coincide on $A^{c}$, she should prefer $f$ to $g$ after observing that $A$ occurred. It is well known that under SEU, both dynamic consistency and consequentialism are satisfied (e.g. see Dominiak and Lefort (2011)). It is also well known that contrary to the Bayes' updating rule for probabilities, the three updating rules for capacities do not satisfy dynamic consistency.

Lemma 3. The Dempster-Shafer, naive Bayes', and Fagin-Halpern updating rules do not necessarily satisfy dynamic consistency. 
Recall Example 2 in the Introduction. Recall also that $v_{A}^{*}$ corresponds to the three updating rules (the Dempster-Shafer, naive Bayes', and Fagin-Halpern updating rules) for a capacity $v$ given $A$. Let $A=\{R, B\}$. If the DM's preferences are captured by a capacity $v$ and the choices in Example 2 are consistent with dynamic consistency, then the following inequalities hold:

$$
\begin{aligned}
& v_{A}^{*}(R)>v_{A}^{*}(B), \text { and } \\
& v_{A}^{*}(R \cup Y)<v_{A}^{*}(B \cup Y) .
\end{aligned}
$$

From Lemma 2, it follows that

$$
\begin{aligned}
& v_{A}^{*}(R)=v_{A}^{*}(R \cup Y), \text { and } \\
& v_{A}^{*}(B)=v_{A}^{*}(B \cup Y) .
\end{aligned}
$$

From Example 2, we find that if both Equalities (4) and (5) hold, then either Inequality (2) or Inequality (3) is violated, which proves Lemma 3 in an informal way. Moreover, from simple calculations, we can also find which updating rules violate Inequality (2) or Inequality (3). If the DM's beliefs are updated by the Dempster-Shafer updating rule, it follows that

$$
\begin{aligned}
& v_{A}^{D S}(R)=v_{A}^{D S}(R \cup Y)=\frac{v(R \cup Y)-v(Y)}{1-v(Y)} \\
< & \frac{v(B \cup Y)-v(Y)}{1-v(Y)}=v_{A}^{D S}(B)=v_{A}^{D S}(B \cup Y),
\end{aligned}
$$

where the inequality holds by Inequality (1). Thus, the Dempster-Shafer updating rule satisfies Inequality (3), but does not satisfy Inequality (2).

If the DM's beliefs are updated by the naive Bayes' updating rule, it follows that

$$
v_{A}^{N B}(R)=v_{A}^{N B}(R \cup Y)=\frac{v(R)}{v(R \cup B)}>\frac{v(B)}{v(R \cup B)}=v_{A}^{N B}(B)=v_{A}^{N B}(B \cup Y),
$$

where the inequality holds by Inequality (1). Thus, the naive Bayes' updating rule satisfies Inequality (2), but does not satisfy Inequality (3).

If the DM's beliefs are updated by the Fagin-Halpern updating rule, it follows that

$$
\begin{aligned}
& v_{A}^{F H}(R)=v_{A}^{F H}(R \cup Y)=\frac{v(R)}{v(R)+1-v(R \cup Y)} \text { and } \\
& v_{A}^{F H}(B)=v_{A}^{F H}(B \cup Y)=\frac{v(B)}{v(B)+1-v(B \cup Y)}
\end{aligned}
$$


Thus, there exist two possibilities that Inequality (2) is satisfied and Inequality (3) is violated, and vice versa.

The above discussions lead to the following observations:

1. While the three updating rules for capacities satisfy consequentialism, the three updating rules do not satisfy dynamic consistency.

2. While the three updating rules for capacities do not fully satisfy dynamic consistency, the three updating rules partially satisfy dynamic consistency.

Therefore, the following viewpoints are worth investigating:

1. What kinds of axioms are satisfied by the updating rules with consequentialism under CEU?

2. To what extent do the updating rules for capacities with consequentialism satisfy dynamic consistency?

The former viewpoint is discussed in Section 3. We discuss the latter viewpoint as follows. Keeping in mind that while the Dempster-Shafer updating rule satisfies Inequality (3), it does not satisfy Inequality (2), we focus on the difference between Inequalities (2) and (3). As in the discussion above, let $A=\{R, B\}$. While acts $f$ and $f^{\prime}$ do not take the maximum value on $A^{c}=\{Y\}, g$ and $g^{\prime}$ take the maximum value on $A^{c}$. From this observation, we propose the notion of upper-constrained dynamic consistency in Section 4.

Similar to the above argument, while acts $f$ and $f^{\prime}$ take the minimum value on $\{R, B\}^{c}=\{Y\}, g$ and $g^{\prime}$ do not take the minimum value on $\{R, B\}^{c}=\{Y\}$. From this observation, we propose the notion of lower-constrained dynamic consistency in Section 4 .

\section{An Axiomatization of C-Capacities}

In this section, we provide an axiomatization of C-capacities within the framework of Schmeidler (1989). First, we introduce the A-conditional comonotonicity that extends the notion of comonotonicity.

Definition 5. Let $x, y \in \mathbb{R}^{\Omega}$. Two functions $x$ and $y$ are comonotonic if $(x(\omega)-$ $\left.x\left(\omega^{\prime}\right)\right)\left(y(\omega)-y\left(\omega^{\prime}\right)\right) \geq 0$ for all $\omega, \omega^{\prime} \in \Omega$.

Definition 6. Let $x, y \in \mathbb{R}^{\Omega}$ and $A \in \mathcal{F}$. Two functions $x$ and $y$ are $A$-conditionally comonotonic if $\left(x(\omega)-x\left(\omega^{\prime}\right)\right)\left(y(\omega)-y\left(\omega^{\prime}\right)\right) \geq 0$ for all $\omega, \omega^{\prime} \in A .^{9}$

\footnotetext{
${ }^{9}$ Jouini and Napp (2004) propose the notion of conditionally comonotonic random variables with respect to some $\sigma$-algebra $\mathcal{G}$. However, our notion of conditional comonotonicity is different from that of Jouini and Napp (2004).
} 
Schmeidler (1989) extends the notion of comonotonicity to $L_{0}$ and considers the DM's preference orders on $L_{0}$ as follows.

Definition 7 (Schmeidler (1989)). Let $f, g \in L_{0}$. Two acts $f$ and $g$ are said to be comonotonic if there are no $\omega, \omega^{\prime} \in \Omega$ such that $f(\omega) \succ f\left(\omega^{\prime}\right)$ and $g\left(\omega^{\prime}\right) \succ g(\omega)$.

Based on Definition 7, we propose the following notion. The notion of $A$-conditional comonotonicity of lotteries requires that two lotteries in $L_{0}$ should be comonotonic on a set $A$.

Definition 8. Let $f, g \in L_{0}$ and $A \in \mathcal{F}$. Two acts $f$ and $g$ are said to be $A$ conditionally comonotonic if there are no $\omega, \omega^{\prime} \in A$ such that $f(\omega) \succ f\left(\omega^{\prime}\right)$ and $g\left(\omega^{\prime}\right) \succ g(\omega)$.

For the axiomatization of C-capacities under CEU, the following axioms are in order. In the following arguments, fix an event $A \in \mathcal{F} \backslash\{\Omega, \emptyset\}$.

Axiom 1 (Weak Order). (a) For all $T \in\{\Omega, A\}$ and all $f, g$ in $L_{0}, f \succeq_{T} g$ or $g \succeq_{T} f$.

(b) For all $T \in\{\Omega, A\}$ and all $f, g, h$ in $L_{0}$, if $f \succeq_{T} g$ and $g \succeq_{T} h$, then $f \succeq_{T} h .^{10}$

Axiom 2 (Conditionally Comonotonic Independence). For all $T \in\{\Omega, A\}$ and every pairwise $T$-conditionally comonotonic (i.e., comonotonic or $A$-conditionally comonotonic) $f, g, h \in L_{0}$ and $\alpha \in(0,1), f \succeq_{T} g$ implies $\alpha f+(1-\alpha) h \succeq_{T}$ $\alpha g+(1-\alpha) h$.

Axiom 3 (Continuity). For all $T \in\{\Omega, A\}$ and all $f, g, h$ in $L_{0}$, if $f \succeq_{T} g$ and $g \succeq_{T} h$, then there exist $\alpha$ and $\beta \in(0,1)$ such that $\alpha f+(1-\alpha) h \succeq_{T} g$ and $g \succeq_{T} \beta f+(1-\beta) h$.

Axiom 4 (Monotonicity). For all $T \in\{\Omega, A\}$ and all $f, g$ in $L_{0}$, if $f(\omega) \succeq_{T} g(\omega)$ on $\Omega$, then $f \succeq_{T} g$.

Axiom 5 (Non-degeneracy). There exist $f, g \in L_{c}$ such that $f \succ_{\Omega} g$.

Axiom 6 (Consistency for Lotteries). For all $y_{1}, y_{2}$ in $L_{c} ; y_{1} \succeq_{A} y_{2} \Leftrightarrow y_{1} \succeq_{\Omega} y_{2}$.

Axiom 7 (Singleton Consequentialism Principle). For all $\omega \in A^{c}$ and all $y_{1}, y_{2}, y_{3} \in L_{c},\left(y_{1},\{\omega\} ; y_{3}, \Omega \backslash\{\omega\}\right) \sim_{A}\left(y_{2},\{\omega\} ; y_{3}, \Omega \backslash\{\omega\}\right)$.

\footnotetext{
${ }^{10} \mathrm{~A}$ binary relation $\succeq_{T}$ is a weak order if and only if $\succ_{T}$ is asymmetric and negatively transitive, whereas a binary relation $\succ_{T}$ is asymmetric if for all $f, g \in L_{0}, f \succ_{T} g \Rightarrow g \succ_{T} f$ and it is negatively transitive if for all $f, g, h \in L_{0}, f \nsucc_{T} g$ and $g \nsucc_{T} h \Rightarrow f \nsucc_{T} h$. For example, see Kreps (1988, p.9).
} 
Some comments are in order. Axioms 1, 3, 4, and 5 are standard in the literature. Axiom 2 is a restriction of the Comonotonic Independence Axiom (Schmeidler (1989)) on a certain event $A$. This axiom leads to $A$-conditionally comonotonic additivity (see the Appendix for the definition) in the representation. Axioms 6 and 7 should be mentioned in detail. Axiom 6 states that the unconditional preference $\succeq$ and conditional preference $\succeq_{A}$ coincide on the set of all constant acts $L_{c}$. Axiom 6 plays a key role in deriving the utility function $u$ for $\succeq$ and $\succeq_{A}$. Axiom 7 is the axiom that imposes consequentialism on the singleton set contained in the complementary set of a certain realized event $A$. In the case of SEU, this axiom guarantees that for every event contained in the complementary set, its probability measure is equal to zero. However, in the case of CEU, this statement does not generally hold true. For example, if $v_{A}$ is a capacity, then neither $v_{A}\left(\left\{\omega_{1}, \omega_{2}\right\}\right)=0$ nor $v_{A}\left(\left\{\omega_{1}, \omega_{3}\right\}\right)=v_{A}\left(\left\{\omega_{3}\right\}\right)$ for $\omega_{1}, \omega_{2} \in A^{c}$ and $\omega_{3} \in A$ is guaranteed by only Axiom 7. In our paper, Axiom 2 permits the DM's representation to be $A$-conditionally comonotonic additive. Then, Axiom 7 together with Axiom 2 guarantees that for every event contained in the complementary set, its capacity is equal to zero.

To compare our result with Schmeidler (1989), we present Schmeidler's (1989) axiomatization theorem.

Theorem 1. (Schmeidler (1989)) A binary relation $\succeq$ defined on $L_{0}$ satisfies Weak Order, Comonotonic Independence, Continuity, Monotonicity, and Non-degeneracy if and only if there exist a unique capacity $v$ on $\mathcal{F}$ and a non-constant affine realvalued function $u$ on $Y$ such that for all $f$ and $g$ in $L_{0}$,

$$
f \succeq g \Leftrightarrow \int_{\Omega} u(f(\omega)) d v(\omega) \geq \int_{\Omega} u(g(\omega)) d v(\omega) .
$$

Now, we can provide an axiomatization of C-capacities.

Theorem 2. Binary relations $\succeq$ and $\succeq_{A}$ defined on $L_{0}$ satisfy Axioms $1-7$ if and only if there exist capacities $v$ and $v_{A}$ on $\mathcal{F}$ and a non-constant affine real-valued function $u$ on $Y$ such that for all $f$ and $g$ in $L_{0}$,

$$
\begin{aligned}
& f \succeq g \Leftrightarrow \int_{\Omega} u(f(\omega)) d v(\omega) \geq \int_{\Omega} u(g(\omega)) d v(\omega) \text { and } \\
& f \succeq_{A} g \Leftrightarrow \int_{\Omega} u(f(\omega)) d v_{A}(\omega) \geq \int_{\Omega} u(g(\omega)) d v_{A}(\omega),
\end{aligned}
$$

where $v_{A}(S)=v_{A}(S \cap A)$ for all $S \in \mathcal{F}$.

Proof. (If part) See the Appendix. 
The only if part is proved in the Appendix using Lemmas 4 and 5 with Lemmas 11-14. In the following, we present only Lemmas 4 and 5. Lemmas 11-14 are presented in the Appendix.

Lemma 4. Let $\succeq$ and $\succeq_{A}$ on $L_{0}$ satisfy Axioms 1-7. Then, there exist capacities $v$ and $v_{A}$ on $\mathcal{F}$ and a non-constant affine real-valued function $u$ on $Y$ such that for all $f$ and $g$ in $L_{0}$,

$$
\begin{aligned}
& f \succeq g \Leftrightarrow \int_{\Omega} u(f(\omega)) d v(\omega) \geq \int_{\Omega} u(g(\omega)) d v(\omega) \text { and } \\
& f \succeq_{A} g \Leftrightarrow \int_{\Omega} u(f(\omega)) d v_{A}(\omega) \geq \int_{\Omega} u(g(\omega)) d v_{A}(\omega) .
\end{aligned}
$$

Proof. See the Appendix.

Lemma 5. Let $v_{A}$ be the capacity in Lemma 4. Let $a, b \in \mathbb{R}^{\Omega}$ be any real-valued functions on $\Omega$. If $a$ and $b$ are $A$-conditionally comonotonic, then $\int_{\Omega}(a+b) d v_{A}=$ $\int_{\Omega} a d v_{A}+\int_{\Omega} b d v_{A}$.

Proof. See the Appendix.

For Lemmas 4 and 5, two papers should be mentioned. Ghirardato et al. (2004, Lemma 1) provide an axiomatization of the invariant biseparable preference based on five axioms. Among these five axioms, four (Weak Order, Archimedean Axiom, Monotonicity, and Non-degeneracy) coincide with Axioms 1, 3, 4, and 5 in this paper. Axiom 2 (Certainty Independence) in Ghirardato et al. (2004) differs from Axiom 2 in this paper. ${ }^{11}$ Ghirardato et al. (2004) propose Comonotonic Ambiguity Neutrality, which states that for all $f, g \in L_{0}$ with $f \sim g$, if $f$ and $g$ are comonotonic, then $(1 / 2) f+(1 / 2) g \sim g$. Ghirardato et al. (2004, Proposition 2) also show that if invariant biseparable preferences satisfy Comonotonic Ambiguity Neutrality, then the DM's preferences are represented by CEU. Note that Axiom 2 in this paper implies both Certainty Independence and Comonotonic Ambiguity Neutrality in Ghirardato et al. (2004). Thus, following the result of Ghirardato et al. (2004, Proposition 2), Lemma 4 in this paper holds.

Asano and Kojima (2015) provide an axiomatization theorem, which states that under a set of axioms, the DM's preferences are represented by some functional

\footnotetext{
${ }^{11}$ Ghirardato et al. (2004) show that if the above-mentioned five axioms are satisfied, then the DM's preferences are represented by a monotonic, constant linear functional. For the definition of monotonicity and constant linearity of functionals, see Ghirardato et al. (2004, p.141).
} 
satisfying $\mathcal{E}$-cominimum additivity. ${ }^{12}$ Set $\mathcal{E}=2^{A}$ in Asano and Kojima (2015). Then, Axiom 6 (E-Cominimum Independence) in Asano and Kojima (2015) coincides with Axiom 2 (Conditionally Comonotonic Independence) in this paper. Moreover, Axioms 1, 3, 4, 5, and 6 in Asano and Kojima (2015) correspond to Axioms 1-5 in this paper. Therefore, it follows from Theorem 2 in Asano and Kojima (2015) that Lemma 4 in this paper and Lemma 2 in Asano and Kojima (2015) imply Lemma 5 in this paper.

\section{Constrained Dynamic Consistency}

In this section, by proposing the notions of lower-constrained dynamic consistency and upper-constrained dynamic consistency, we investigate the extent to which the updating rules for capacities with consequentialism satisfy dynamic consistency.

As pointed out in Section 2, the Dempster-Shafer and naive Bayes' updating rules do not satisfy dynamic consistency. According to Machina (1989), dynamic consistency should be satisfied but it is a strong requirement. Thus, by proposing weaker axioms than dynamic consistency, we investigate the extent to which the Dempster-Shafer and naive Bayes' updating rules satisfy dynamic consistency. We first consider the Dempster-Shafer updating rule. Let $f, g \in L_{0}$ and $A \in \mathcal{F} \backslash\{\Omega, \emptyset\}$. Define the subset $\Omega^{U C}(f, g ; A)$ of $\Omega$ by

$$
\begin{array}{r}
\Omega^{U C}(f, g ; A)=A^{c} \cup\left\{\omega \in A \mid\left(\exists \omega^{\prime} \in A^{c}, f(\omega) \succ f\left(\omega^{\prime}\right)\right)\right. \text { or } \\
\left.\left(\exists \omega^{\prime} \in A^{c}, g(\omega) \succ g\left(\omega^{\prime}\right)\right)\right\} .
\end{array}
$$

That is, this set $\Omega^{U C}(f, g ; A)$ is defined by the union of $A^{c}$ and the states in $A$ such that at least either $f$ or $g$ takes values strictly preferred to the minimal values of $f$ or $g$ on $A^{c}$. Applying this notion to Example 2 in the Introduction, we obtain the following:

$$
\Omega^{U C}\left(f, f^{\prime} ;\{R, B\}\right)=\{R, B, Y\} \text { and } \Omega^{U C}\left(g, g^{\prime} ;\{R, B\}\right)=\{Y\} .
$$

Based on $\Omega^{U C}$, we propose the following axiom.

Axiom 8 (Upper-Constrained Dynamic Consistency (UCDC)). Let $A \in \mathcal{F} \backslash\{\Omega, \emptyset\}$ be an event. For all $f$ and $g$ such that $f(\omega)=g(\omega)$ for all $\omega \in$ $\Omega^{U C}(f, g ; A), f \succeq g \Leftrightarrow f \succeq_{A} g$.

\footnotetext{
${ }^{12}$ Let $\mathcal{E} \subseteq 2^{\Omega}$ be a collection of the subsets of $\Omega$. Two functions on $\Omega$ are $\mathcal{E}$-cominimum if, for each $E \in \mathcal{E}$, the set of minimizers of $x$ on $E$ and that of $y$ on $E$ have a common element. A functional $I$ on the set of functions of $\Omega$ is $\mathcal{E}$-cominimum additive if $I(x+y)=I(x)+I(y)$ whenever two functions $x$ and $y$ are $\mathcal{E}$-cominimum. The notion of $\mathcal{E}$-cominimum additivity was first proposed by Kajii et al. (2007).
} 
Applying this axiom to Example 2 in the Introduction, it follows that $g^{\prime} \succ_{A} g$ because $g^{\prime} \succ g$ and $g=g^{\prime}$ on $\Omega^{U C}\left(g, g^{\prime} ; A\right)$, where $A=\{R, B\}$. However, Axiom 8 does not require $f^{\prime} \succ_{A} f$. The following lemma shows that the Dempster-Shafer updating rule satisfies Axiom 8.

Lemma 6. Let $A \in \mathcal{F} \backslash\{\Omega, \emptyset\}$ be an event. Suppose that unconditional and conditional preferences, $\succeq$ and $\succeq_{A}$ on $L_{0}$, are captured by a capacity $v$ and its DempsterShafer updating rule $v_{A}^{D S}$, respectively. Moreover, suppose that $v\left(A^{c}\right) \neq 1$. Then, such preferences, $\succeq$ and $\succeq_{A}$, satisfy Axiom 8 .

Proof. See the Appendix.

Conversely, if the DM's preferences are captured by a C-capacity and satisfy Axiom 8, her conditional capacity is equal to the Dempster-Shafer updating rule.

Lemma 7. Let $A \in \mathcal{F} \backslash\{\Omega, \emptyset\}$ be an event. Suppose that unconditional and conditional preferences, $\succeq$ and $\succeq_{A}$ on $L_{0}$, are captured by a capacity $v$ and its $C$-capacity $v_{A}$, respectively. Moreover, suppose that $v\left(A^{c}\right) \neq 1$. Then, if $\succeq$ and $\succeq_{A}$ satisfy Axiom 8, $v_{A}=v_{A}^{D S}$.

The next corollary follows from Lemma 7 and Theorem 2.

Corollary 2. Let $A \in \mathcal{F} \backslash\{\Omega, \emptyset\}$ be an event. Suppose that unconditional and conditional preferences, $\succeq$ and $\succeq_{A}$ on $L_{0}$, satisfy Axioms 1-7, and Axiom 8. For the derived capacity $v$, if $v(A) \neq 1$, the derived conditional $C$-capacity $v_{A}$ is equal to $v_{A}^{D S}$.

Lemma 7 can be shown by Axiom 8 and the following axiom (Axiom GS1). Gilboa and Schmeidler (1993) provide a choice-theoretic foundation with the DempsterShafer updating rule.

\section{Axiom GS1 (Gilboa and Schmeidler (1993)).}

$f \succeq_{A} g \Leftrightarrow\left(f, A ; y^{*}, A^{c}\right) \succeq\left(g, A ; y^{*}, A^{c}\right)$, where $y^{*}$ is the maximal outcome.

If the DM satisfies Axiom GS1, she is assumed to consider that the maximal (or the best) outcome would have followed if an event $A$ had not occurred. That is, she considers that the maximal outcome has occurred in $A^{c}$. In this sense, the Dempster-Shafer updating rule is a pessimistic inference.

Axiom GS1 is a superior axiom in that it fully captures the characteristics of the Dempster-Shafer updating rule by only one axiom. However, from the viewpoint 
of dynamic consistency, there exists some room for improvement. First, in Axiom GS1, the DM's conditional preferences are assumed to compare two acts in which the outcomes are constant, $y^{*}$, in $A^{c}$, which is considered to be a strong requirement. Second, the relation between dynamic consistency and Axiom GS1 is blurred because the acts in the DM's preferences with respect to the conditional preferences $\succeq_{A}$ are different from those with respect to the unconditional preferences $\succeq$. By introducing upper-constrained dynamic consistency, we can clarify that Axiom GS1 simultaneously captures two behavioral features; that is, Axiom GS1 holds if and only if both consequentialism and Axiom 8 (UCDC) hold.

First, we assume consequentialism and Axiom 8. We define two acts $F$ and $G$ by $F=\left(f, A ; y^{*}, A^{c}\right)$ and $G=\left(g, A ; y^{*}, A^{c}\right)$, respectively. Suppose that $F \succeq G$. Because $\Omega^{U C}(F, G ; A)=A^{c}, F(\omega)=G(\omega)$ for all $\omega \in \Omega^{U C}(F, G ; A)$. It follows from Axiom 8 that $F \succeq G \Leftrightarrow F \succeq_{A} G$. It also follows from consequentialism that $F \sim_{A} f$ and $G \sim_{A} g$. Then, $F \succeq G \Leftrightarrow f \succeq_{A} g$, which is exactly GS1. We show the converse in Section 5 .

Now, we turn to the naive Bayes' updating rule. Let $f, g \in L_{0}$ and $A \in \mathcal{F} \backslash\{\Omega, \emptyset\}$. Define the subset $\Omega^{L C}(f, g ; A)$ of $\Omega$ by

$$
\begin{array}{r}
\Omega^{L C}(f, g ; A)=A^{c} \cup\left\{\omega \in A \mid\left(\exists \omega^{\prime} \in A^{c}, f\left(\omega^{\prime}\right) \succ f(\omega)\right)\right. \text { or } \\
\left.\left(\exists \omega^{\prime} \in A^{c}, g\left(\omega^{\prime}\right) \succ g(\omega)\right)\right\} .
\end{array}
$$

That is, this set $\Omega^{L C}(f, g ; A)$ is defined by the union of $A^{c}$ and the states in $A$ such that at least either $f$ or $g$ takes values strictly less preferred to the maximal values of $f$ or $g$ on $A^{c}$. Applying this notion to Example 2 in the Introduction, we obtain the following:

$$
\Omega^{L C}\left(f, f^{\prime} ;\{R, B\}\right)=\{Y\} \text { and } \Omega^{L C}\left(g, g^{\prime} ;\{R, B\}\right)=\{R, B, Y\} .
$$

Based on $\Omega^{L C}$, we propose the following axiom.

Axiom 9 (Lower-Constrained Dynamic Consistency (LCDC)). Let $A \in \mathcal{F} \backslash\{\Omega, \emptyset\}$ be an event. For all $f$ and $g$ such that $f(\omega)=g(\omega)$ for all $\omega \in$ $\Omega^{L C}(f, g ; A), f \succeq g \Leftrightarrow f \succeq_{A} g$.

Applying this axiom to Example 2 in the Introduction, it follows that $f \succ_{A} f^{\prime}$ because $f \succ f^{\prime}$ and $f=f^{\prime}$ on $\Omega^{L C}\left(f, f^{\prime} ; A\right)$, where $A=\{R, B\}$. However, Axiom 9 does not require $g \succ_{A} g^{\prime}$. The following lemma shows that the naive Bayes' updating rule satisfies Axiom 9. 
Lemma 8. Let $A \in \mathcal{F} \backslash\{\Omega, \emptyset\}$ be an event. Suppose that unconditional and conditional preferences, $\succeq$ and $\succeq_{A}$ on $L_{0}$, are captured by a capacity $v$ and its naive Bayes' updating rule $v_{A}^{N B}$, respectively. Moreover, suppose that $v(A) \neq 0$. Then, such preferences, $\succeq$ and $\succeq_{A}$, satisfy Axiom 9.

Proof. We omit the proof because this lemma can be shown in a similar way to the proof of Lemma 6.

Conversely, if the DM's preferences are captured by a C-capacity and satisfy Axiom 9, her conditional capacity is equal to the naive Bayes' updating rule.

Lemma 9. Let $A \in \mathcal{F} \backslash\{\Omega, \emptyset\}$ be an event. Suppose that unconditional and conditional preferences, $\succeq$ and $\succeq_{A}$ on $L_{0}$, are captured by a capacity $v$ and its $C$-capacity $v_{A}$, respectively. Moreover, suppose that $v(A) \neq 0$. Then, if $\succeq$ and $\succeq_{A}$ satisfy Axiom 9, $v_{A}=v_{A}^{N B}$.

The next corollary follows from Lemma 9 and Theorem 2.

Corollary 3. Let $A \in \mathcal{F} \backslash\{\Omega, \emptyset\}$ be an event. Suppose that unconditional and conditional preferences, $\succeq$ and $\succeq_{A}$ on $L_{0}$, satisfy Axioms 1-7, and Axiom 9. For the derived capacity $v$, if $v(A) \neq 0$, then the derived conditional $C$-capacity $v_{A}$ is equal to $v_{A}^{N B}$.

It is worth investigating the relationship between Axiom 9 and the following axiom proposed by Gilboa and Schmeidler (1993). Gilboa and Schmeidler (1993) provide a choice-theoretic foundation with the naive Bayes' updating rule.

\section{Axiom GS2 (Gilboa and Schmeidler (1993)).}

$f \succeq_{A} g \Leftrightarrow\left(f, A ; y_{*}, A^{c}\right) \succeq\left(g, A ; y_{*}, A^{c}\right)$, where $y_{*}$ is the minimal outcome. ${ }^{13}$

An argument similar to Axiom GS1 can be applied to Axiom GS2, and it can be shown that both consequentialism and Axiom 9 (LCDC) imply Axiom GS2; hence, the results in Section 5 show the converse. As a result, we can show that Axiom GS2 holds if and only if both consequentialism and Axiom 9 hold.

\section{Mean-Preserving Axiom and Three Updating Rules}

\footnotetext{
${ }^{13}$ If the DM satisfies Axiom GS2, she is assumed to consider that the minimal (or the worst) outcome would have followed if an event $A$ had not occurred. That is, she considers that the minimal outcome has occurred in $A^{c}$. In this sense, the naive Bayes' updating rule is an optimistic one.
} 
In Sections 3 and 4, C-capacities played a significant role in axiomatizations. Section 3 provided an axiomatization of C-capacities. Based on C-capacities, Section 4 provided axiomatizations of the Dempster-Shafer and naive Bayes' updating rules by UCDC and LCDC. In a related paper, Eichberger et al. (2007) provide an axiomatization of the Fagin-Halpern updating rule based on consequentialism. In the spirit of Eichberger et al. (2007), this section provides a unified approach for distinguishing capacity updating rules according to the degree of dynamic consistency. For this purpose, we introduce the notion of the mean-preserving rule (MPR) as axioms.

The purpose of introducing the MPR is threefold. First, through the MPR, we can show that Axiom GS1 implies Axiom 8 (UCDC) and consequentialism, which shows that Axiom GS1 holds if and only if both Axiom 8 (UCDC) and consequentialism hold. Similarly, through the MPR, we can show that Axiom GS2 implies Axiom 9 (LCDC) and consequentialism, which shows that Axiom GS2 holds if and only if both Axiom 9 (LCDC) and consequentialism. ${ }^{14}$ Second, we can also axiomatize the Fagin-Halpern updating rule. Finally, we can characterize the three updating rules for capacities within the same framework (i.e., the MPR), which enables us to illuminate the properties of the three updating rules from a consistent viewpoint.

First, to introduce the MPR, we present the following lemma, which is easily shown. Therefore, the proof is omitted.

Lemma 10. Let $A \in \mathcal{F} \backslash\{\Omega, \emptyset\}$ be an event. Let $\succeq$ and $\succeq_{A}$ be captured by a probability $p$, its conditional probability $p_{A}$, and a common utility function $u$. Suppose that $p(A) \neq 0$. Then, for any $f \in L_{0}$ and any $m \in L_{c}$,

$$
f \sim\left(m, A ; f, A^{c}\right) \Leftrightarrow f \sim_{A}\left(m, A ; f, A^{c}\right)
$$

Furthermore, it holds that

$$
u(m)=\int_{A} u\left(\left.f\right|_{A}\right) d p_{A}
$$

We call this property, including Equation (6), the MPR. This rule states that for unconditional and conditional preferences, we can take any constant act $m$ that makes $f$ indifferent on an event $A$, which can be interpreted as some kind of averaging. The MPR, which holds for SEU, does not necessarily hold under CEU. This is because the rank-dependent property of $\mathrm{CEU}$ makes $\mathrm{CEU}$ violate the sure-thing

\footnotetext{
${ }^{14}$ Recall that in Section 4, we showed that Axiom 8 and consequentialism imply Axiom GS1 and that Axiom 9 and consequentialism imply Axiom GS2.
} 
principle. However, by restricting acts extremely, a slightly modified MPR holds for the three updating rules for capacities, and these updating rules are axiomatized under the MPR.

First, we axiomatize the Dempster-Shafer updating rule. Let $y^{*}$ and $y_{*}$ be the maximal and minimal outcomes, respectively. Let $A \in \mathcal{F} \backslash\{\Omega, \emptyset\}$ be an event. Define the set of acts, $L_{y^{*}, A^{c}}^{2} \subset L_{0}$, whose elements satisfy the following:

$$
f(\omega)=\left\{\begin{array}{cc}
y^{*} \text { or } y_{*} & \text { if } \omega \in A \\
y^{*} & \text { if } \omega \in A^{c}
\end{array}\right.
$$

For axiomatizing the Dempster-Shafer updating rule, we propose the following axiom that imposes the MPR only on $L_{y^{*}, A^{c}}^{2}$.

Axiom 10. Let $A \in \mathcal{F} \backslash\{\Omega, \emptyset\}$ be an event. For all $f \in L_{y^{*}, A^{c}}^{2}$, and all $m \in L_{c}$,

$$
f \sim\left(m, A ; f, A^{c}\right) \Leftrightarrow f \sim_{A}\left(m, A ; f, A^{c}\right) .
$$

Based on Axiom 10, the Dempster-Shafer updating rule can be axiomatized as follows.

Theorem 3. Let $A \in \mathcal{F} \backslash\{\Omega, \emptyset\}$ be an event. Let $\succeq$ and $\succeq_{A}$ be captured by a capacity $v$, its $C$-capacity $v_{A}$, and a common utility function $u$. Suppose that $v\left(A^{c}\right) \neq 1$. Then, the following are equivalent.

(i) A conditional preference $\succeq_{A}$ satisfies Axiom 10.

(ii) $v_{A}=v_{A}^{D S}$.

Proof. Note that $1-v\left(A^{c}\right) \neq 0$. Take any $S \in \mathcal{F}$, and define $f_{S} \in L_{y^{*}, A^{c}}^{2}$ as follows:

$$
f_{S}(\omega)=\left\{\begin{array}{lll}
y^{*} & \text { if } \quad \omega \in S \cup A^{c} \\
y_{*} & \text { if } \quad \omega \in S^{c} \cap A
\end{array}\right.
$$

Then, it follows that

$$
\begin{aligned}
& f_{S} \sim\left(m, A ; f_{S}, A^{c}\right) \\
\Leftrightarrow & \left(u\left(y^{*}\right)-u\left(y_{*}\right)\right) v\left(S \cup A^{c}\right)+u\left(y_{*}\right)=\left(u\left(y^{*}\right)-u(m)\right) v\left(A^{c}\right)+u(m) \\
\Leftrightarrow & \left(u\left(y^{*}\right)-u\left(y_{*}\right)\right) v\left(S \cup A^{c}\right)-\left(u\left(y^{*}\right)-u\left(y_{*}\right)\right) v\left(A^{c}\right)+u\left(y_{*}\right) \\
= & \left(u\left(y^{*}\right)-u(m)\right) v\left(A^{c}\right)-\left(u\left(y^{*}\right)-u\left(y_{*}\right)\right) v\left(A^{c}\right)+u(m) \\
\Leftrightarrow & \left(u\left(y^{*}\right)-u\left(y_{*}\right)\right)\left(v\left(S \cup A^{c}\right)-v\left(A^{c}\right)\right)=\left(u(m)-u\left(y_{*}\right)\right)\left(1-v\left(A^{c}\right)\right) \\
\Leftrightarrow & \frac{u(m)-u\left(y_{*}\right)}{u\left(y^{*}\right)-u\left(y_{*}\right)}=\frac{v\left(S \cup A^{c}\right)-v\left(A^{c}\right)}{1-v\left(A^{c}\right)}=v_{A}^{D S}(S) .
\end{aligned}
$$


Similarly, it follows that

$$
\begin{aligned}
& f_{S} \sim_{A}\left(m, A ; f_{S}, A^{c}\right) \\
\Leftrightarrow & \frac{u(m)-u\left(y_{*}\right)}{u\left(y^{*}\right)-u\left(y_{*}\right)}=\frac{v_{A}\left(S \cup A^{c}\right)-v_{A}\left(A^{c}\right)}{1-v_{A}\left(A^{c}\right)}=v_{A}(S),
\end{aligned}
$$

where the last equality holds by $v_{A}\left(S \cup A^{c}\right)=v_{A}\left(\left(S \cup A^{c}\right) \cap A\right)=v_{A}(S \cap A)=v_{A}(S)$ and $v_{A}\left(A^{c}\right)=v_{A}\left(A^{c} \cap A\right)=0$ since $v_{A}$ is a C-capacity. If Axiom 10 holds, $v_{A}=v_{A}^{D S}$ by Equations (7) and (8). Conversely, suppose that $v_{A}=v_{A}^{D S}$. Take any $f \in L_{y^{*}, A^{c}}^{2}$. Let $S=\left\{\omega \in A \mid f(\omega)=y^{*}\right\}$. Then, $f_{S}$ coincides with $f$. Since $v_{A}=v_{A}^{D S}$, Axiom 10 holds by Equations (7) and (8).

We next investigate the relationship between Axiom GS1 and Axiom 10. First, we can check that Axiom GS1 implies Axiom 10 and consequentialism as follows. Axiom GS1 implies Axiom 10 because $f_{S}(\omega)=y^{*}$ on $A^{c}$. Next, let $f, g \in L_{0}$ with $\left.f\right|_{A}=\left.g\right|_{A}$. Then, $\left(f, A ; y^{*}, A^{c}\right) \sim\left(g, A ; y^{*}, A^{c}\right)$ since $\left(f, A ; y^{*}, A^{c}\right)=\left(g, A ; y^{*}, A^{c}\right)$. If Axiom GS1 holds, $f \sim_{A} g$, which shows that Axiom GS1 implies consequentialism. Then, we know the following implications:

the Dempster-Shafer updating rule $\Rightarrow$ Axiom 8 (UCDC) + consequentialism $\Rightarrow$ Axiom GS1 $\Rightarrow$ Axiom $10+$ consequentialism $\Rightarrow$ the Dempster-Shafer updating rule.

The first and second implications follow from the argument in Section 4, the third implication follows from the above argument, and the fourth implication (indeed, the equivalence) follows from Theorem 3. Therefore, we obtain the following equivalences:

the Dempster-Shafer updating rule $\Leftrightarrow$ Axiom 8 (UCDC) + consequentialism $\Leftrightarrow$ GS1 $\Leftrightarrow$ Axiom $10+$ consequentialism.

Next, we axiomatize the naive Bayes' updating rule. Let $y^{*}$ and $y_{*}$ be the maximal and minimal outcomes, respectively. Let $A \in \mathcal{F} \backslash\{\Omega, \emptyset\}$ be an event. Define the set of acts, $L_{y_{*}, A^{c}}^{2} \subset L_{0}$, whose elements satisfy the following:

$$
f(\omega)=\left\{\begin{array}{ccc}
y^{*} \text { or } y_{*} & \text { if } \quad \omega \in A \\
y_{*} & \text { if } \omega \in A^{c} .
\end{array}\right.
$$

For axiomatizing the naive Bayes' updating rule, we propose the following axiom that imposes the MPR only on $L_{y_{*}, A^{c}}^{2}$.

Axiom 11. Let $A \in \mathcal{F} \backslash\{\Omega, \emptyset\}$ be an event. For all $f \in L_{y_{*}, A^{c}}^{2}$, and all $m \in L_{c}$,

$$
f \sim\left(m, A ; f, A^{c}\right) \Leftrightarrow f \sim_{A}\left(m, A ; f, A^{c}\right) .
$$

Based on Axiom 11, the naive Bayes' updating rule can be axiomatized as follows. 
Theorem 4. Let $A \in \mathcal{F} \backslash\{\Omega, \emptyset\}$ be an event. Let $\succeq$ and $\succeq_{A}$ be captured by a capacity $v$, its $C$-capacity $v_{A}$, and a common utility function $u$. Suppose that $v(A) \neq 0$. Then, the following are equivalent.

(i) A conditional preference $\succeq_{A}$ satisfies Axiom 11.

(ii) $v_{A}=v_{A}^{N B}$.

Proof. Note that $v(A) \neq 0$. Take any $S \in \mathcal{F}$, and define $f_{S} \in L_{y_{*}, A^{c}}^{2}$ as follows:

$$
f_{S}(\omega)= \begin{cases}y^{*} & \text { if } \omega \in S \cap A \\ y_{*} & \text { otherwise }\end{cases}
$$

Then, it follows that

$$
f_{S} \sim\left(m, A ; f_{S}, A^{c}\right) \Leftrightarrow \frac{u(m)-u\left(y_{*}\right)}{u\left(y^{*}\right)-u\left(y_{*}\right)}=\frac{v(S \cap A)}{v(A)}=v_{A}^{N B}(S) .
$$

Furthermore, similarly, it follows that

$$
f_{S} \sim_{A}\left(m, A ; f_{S}, A^{c}\right) \Leftrightarrow \frac{u(m)-u\left(y_{*}\right)}{u\left(y^{*}\right)-u\left(y_{*}\right)}=v_{A}(S) .
$$

Therefore, if Axiom 11 holds, $v_{A}=v_{A}^{N B}$. Similar to the proof of Theorem 3, the converse can be proved.

As with the Dempster-Shafer updating rule, we can show that Axiom GS2 implies both Axiom 11 and consequentialism. ${ }^{15}$ Then, we know the following implications: the naive Bayes' updating rule $\Rightarrow$ Axiom 9 (LCDC) + consequentialism $\Rightarrow$ Axiom GS2 $\Rightarrow$ Axiom $11+$ consequentialism $\Rightarrow$ the naive Bayes' updating rule.

The first and second implications follow from the argument in Section 4, the third implication follows from the above argument, and the fourth implication (indeed, the equivalence) follows from Theorem 4. Therefore, we obtain the following equivalences:

the naive Bayes' updating rule $\Leftrightarrow$ Axiom 9 (LCDC) + consequentialism $\Leftrightarrow$ GS2 $\Leftrightarrow$ Axiom $11+$ consequentialism.

Finally, based on C-capacities, we axiomatize the Fagin-Halpern updating rule as well as the Dempster-Shafer and naive Bayes' updating rules. Let $m \in L_{c}$ such that $y^{*} \succ m \succ y_{*}$, where $y^{*}$ and $y_{*}$ be the maximal and minimal outcomes, respectively. Let $A \in \mathcal{F} \backslash\{\Omega, \emptyset\}$ be an event. Define the set of acts, $L_{m, A^{c}}^{3} \subset L_{0}$, whose elements satisfy the following:

$$
f(\omega)=\left\{\begin{array}{ccc}
y^{*} \text { or } y_{*} & \text { if } \omega \in A \\
m & \text { if } \omega \in A^{c} .
\end{array}\right.
$$

\footnotetext{
${ }^{15}$ Note that Axiom GS2 implies Axiom 11 because $f_{S}(\omega)=y_{*}$ on $A^{c}$. Next, let $f, g \in L_{0}$ with $\left.f\right|_{A}=\left.g\right|_{A}$. Then, $\left(f, A ; y_{*}, A^{c}\right) \sim\left(g, A ; y_{*}, A^{c}\right)$ since $\left(f, A ; y_{*}, A^{c}\right)=\left(g, A ; y_{*}, A^{c}\right)$.
} 
For axiomatizing the Fagin-Halpern updating rule, we propose the following axiom that imposes the MPR only on $L_{m, A^{c}}^{3}$.

Axiom 12. Let $A \in \mathcal{F} \backslash\{\Omega, \emptyset\}$ be an event. For all $m \in L_{c}$ such that $y^{*} \succ m \succ y_{*}$, and all $f \in L_{m, A^{c}}^{3}$,

$$
f \sim\left(m, A ; f, A^{c}\right) \Leftrightarrow f \sim_{A}\left(m, A ; f, A^{c}\right)
$$

Note that $\left(m, A ; f, A^{c}\right)=\left(m, A ; m, A^{c}\right)=m$. Therefore, Axiom 12 states that $f \sim m \Leftrightarrow f \sim_{A} m$. Based on Axiom 12, the Fagin-Halpern updating rule can be axiomatized as follows.

Theorem 5. Let $A \in \mathcal{F} \backslash\{\Omega, \emptyset\}$ be an event. Let $\succeq$ and $\succeq_{A}$ be captured by a capacity $v$, its $C$-capacity $v_{A}$, and a common utility function $u$. Suppose that $v(S \cap A)+1-$ $v\left(S \cup A^{c}\right) \neq 0$ for all $S \in \mathcal{F}$. Then, the following are equivalent.

(i) A conditional preference $\succeq_{A}$ satisfies Axiom 12.

(ii) $v_{A}=v_{A}^{F H}$.

Proof. Note that $v(S \cap A)+1-v\left(S \cup A^{c}\right) \neq 0$. Take any $S \in \mathcal{F} \backslash\{\Omega, \emptyset\}$, and define $f_{S} \in L_{m, A^{c}}^{3}$ as follows:

$$
f_{S}(\omega)=\left\{\begin{array}{lll}
y^{*} & \text { if } & \omega \in S \cap A \\
y_{*} & \text { if } & \omega \in S^{c} \cap A \\
m & \text { if } & \omega \in A^{c} .
\end{array}\right.
$$

Then, it follows that

$$
\begin{aligned}
& f_{S} \sim m \\
\Leftrightarrow & \left(u\left(y^{*}\right)-u(m)\right) v(S \cap A)+\left(u(m)-u\left(y_{*}\right)\right) v\left(S \cup A^{c}\right)+u\left(y_{*}\right)=u(m) \\
\Leftrightarrow & u\left(y^{*}\right) v(S \cap A)-u\left(y_{*}\right) v\left(S \cup A^{c}\right)+u\left(y_{*}\right) \\
= & u(m)\left(v(S \cap A)+1-v\left(S \cup A^{c}\right)\right) \\
\Leftrightarrow & \left(u\left(y^{*}\right)-u\left(y_{*}\right)\right) v(S \cap A) \\
= & \left(u(m)-u\left(y_{*}\right)\right)\left(v(S \cap A)+1-v\left(S \cup A^{c}\right)\right) \\
\Leftrightarrow & \frac{u(m)-u\left(y_{*}\right)}{u\left(y^{*}\right)-u\left(y_{*}\right)}=\frac{v(S \cap A)}{v(S \cap A)+1-v\left(S \cup A^{c}\right)}=v_{A}^{F H}(S) .
\end{aligned}
$$

Since $v_{A}(S)=v_{A}(S \cap A)$ by the assumption that $v_{A}$ is a C-capacity, similar to the above, it follows that

$$
f_{S} \sim_{A} m
$$




$$
\begin{aligned}
& \Leftrightarrow \frac{u(m)-u\left(y_{*}\right)}{u\left(y^{*}\right)-u\left(y_{*}\right)} \\
& =\frac{v_{A}(S \cap A)}{v_{A}(S \cap A)+1-v_{A}\left(S \cup A^{c}\right)} \\
& =\frac{v_{A}(S)}{v_{A}(S)+1-v_{A}(S)}=v_{A}(S) .
\end{aligned}
$$

Therefore, if Axiom 12 holds, $v_{A}=v_{A}^{F H}$. Similar to the proof of Theorem 3, the converse can be proved.

It is worth mentioning the relationship between Eichberger et al. (2007) and this paper. Based on three axioms (Consequentialism, State Independence, and Conditional Certainty Equivalent Consistency (CCEC)), Eichberger et al. (2007) showed that the DM's conditional preferences are captured by the Fagin-Halpern updating rule. Since the axiom restricting sets of acts $\mathcal{F}$ to $L_{A^{c}, m}^{3}$ and $x=m$ in CCEC corresponds to Axiom 12, it holds that CCEC implies Axiom 12. Thus, the implication (i) $\Rightarrow$ (ii) of Theorem 1 in Eichberger et al. (2007) is obtained from Theorem 5. However, as pointed out by Horie (2013), the DM's conditional preferences captured by the Fagin-Halpern updating rule do not satisfy CCEC. By restricting the $\operatorname{acts}^{16}$ to $f \in \mathcal{F}^{2}$ for which CCEC holds, Horie (2013) showed that the converse also holds. Contrary to Horie (2013), this paper obtained the equivalence by restricting the acts to $f \in L_{A^{c}, m}^{3}$ and $x=m$. Our contribution to the literature is threefold. First, this paper provided an axiomatization of the Fagin-Halpern updating rule within the framework of dynamic consistency. Second, this paper provided a unified approach for the three capacity updating rules (the Dempster-Shafer updating rule, naive Bayes' updating rule, and Fagin-Halpern updating rule) according to the degree of dynamic consistency. Finally, the constant act $m$ was defined endogenously. Additionally, by considering the condition $\left(f, A ; \mu, A^{c}\right) \sim\left(m, A ; \mu, A^{c}\right) \Leftrightarrow\left(f, A ; \mu, A^{c}\right) \sim_{A}\left(m, A ; \mu, A^{c}\right)$ with $f(\omega)=y^{*}$ or $y_{*}$ and $\mu \in\left\{y^{*}, m, y_{*}\right\}$ as a condition of dynamic consistency restricted to particular sets of acts $f,{ }^{17}$ we can provide axiomatizations of the Fagin-Halpern updating rule, Dempster-Shafer updating rule, and naive Bayes' updating rule by some kind of constrained dynamic consistency.

\section{Conclusion}

\footnotetext{
$\left.2^{\Omega}\right\}$.

${ }^{16}$ Horie (2013) defines the set of binary acts $\mathcal{F}^{2}$ by $\left\{\left(b, A ; \omega, A^{c}\right) \mid b, w \in X\right.$ such that $b \geq w$ and $A \in$

${ }^{17} \mathrm{We}$ acknowledge an anonymous reviewer who points out this condition.
} 
By proposing the notions of upper-constrained dynamic consistency and lowerconstrained dynamic consistency that are weaker axioms than dynamic consistency, this paper axiomatized the Dempster-Shafer updating rule and naive Bayes' updating rule under CEU. Based on the notion of conditional comonotonicity, this paper also provided an axiomatization of consequentialism under CEU. Furthermore, based on the notion of the MPR, this paper provided a unified approach for distinguishing capacity updating rules (the Dempster-Shafer updating rule, naive Bayes' updating rule, and Fagin-Halpern updating rule) according to the degree of dynamic consistency. 


\section{Appendix}

Definition 9. An operator $I: \mathbb{R}^{\Omega} \rightarrow \mathbb{R}$ is comonotonic additive if $I(x+y)=$ $I(x)+I(y)$ whenever $x$ and $y$ are comonotonic.

It is well known that Choquet integrals satisfy comonotonic additivity. Furthermore, Schmeidler (1986) shows that if an operator $I: \mathbb{R}^{\Omega} \rightarrow \mathbb{R}$ satisfies comonotonic additivity and monotonicity (i.e., $x \geq y$ on $\Omega$ implies $I(x) \geq I(y)$ for all $x, y \in \mathbb{R}^{\Omega}$ ), then $I$ is a Choquet integral. As mentioned in Schmeidler (1986), if an operator $I: \mathbb{R}^{\Omega} \rightarrow \mathbb{R}$ satisfies comonotonic additivity and monotonicity, then it satisfies positive homogeneity of degree 1 (that is, $I(t x)=t I(x)$ for all $t \geq 0$ ). Moreover, it is shown that if an operator $I: \mathbb{R}^{\Omega} \rightarrow \mathbb{R}$ satisfies comonotonic additivity and positive homogeneity of degree 1 , then $I$ is a Choquet integral.

Definition 10. Choquet integrals with respect to a capacity $v$ are said to be $A$ conditionally comonotonic additive if $\int_{\Omega}(x+y) d v=\int_{\Omega} x d v+\int_{\Omega} y d v$ whenever $x$ and $y$ are $A$-conditionally comonotonic.

For $A$-conditional comonotonicity, we can show the following proposition.

Proposition 2. Let $A \in \mathcal{F}$. Let $v$ be a capacity such that $v(S \cap A)=v(S)$ for any $S \in \mathcal{F}$. Then, the Choquet integral of $x$ with respect to such a capacity $v, \int_{\Omega} x d v$, is A-conditionally comonotonic additive. That is, $\int_{\Omega}(x+y) d v=\int_{\Omega} x d v+\int_{\Omega} y d v$ if $x$ and $y$ are A-conditionally comonotonic. Moreover, $\int_{\Omega} x d v=\left.\left.\int_{A} x\right|_{A} d v\right|_{A}$ where $\left.x\right|_{A}$ and $\left.v\right|_{A}$ are the restriction of a function $x$ and the restriction of a capacity $v$ on a set $A$, respectively.

Proof. Let $\left\langle\omega_{1}, \omega_{2}, \cdots, \omega_{n}\right\rangle$ be a permutation of all the elements of $\Omega$ satisfying $x\left(\omega_{1}\right) \geq x\left(\omega_{2}\right) \geq \cdots \geq x\left(\omega_{n}\right)$. Moreover, let $A=\left\{\omega_{i(1)}, \omega_{i(2)}, \cdots, \omega_{i(k)}\right\}$, where $\{i(1), i(2), \cdots, i(k)\} \subseteq\{1,2, \cdots n\}$ and $i(1)<i(2)<\cdots<i(k)$ for $k=|A|$. Then,

$$
\begin{aligned}
& \int_{\Omega} x(\omega) d v \\
= & \sum_{1 \leq p \leq n-1}\left(x\left(\omega_{p}\right)-x\left(\omega_{p+1}\right)\right) v\left(\left\{\omega_{1}, \ldots, \omega_{p}\right\}\right)+x\left(\omega_{n}\right) v(\Omega) \\
= & \sum_{1 \leq p \leq n-1}\left(x\left(\omega_{p}\right)-x\left(\omega_{p+1}\right)\right) v\left(\left\{\omega_{1}, \ldots, \omega_{p}\right\} \cap A\right)+x\left(\omega_{n}\right) v(\Omega \cap A) \\
= & \sum_{1 \leq p<i(1)}\left(x\left(\omega_{p}\right)-x\left(\omega_{p+1}\right)\right) v(\emptyset) \\
+ & \sum_{1 \leq q \leq k-1}\left(\sum_{i(q) \leq p<i(q+1)}\left(x\left(\omega_{p}\right)-x\left(\omega_{p+1}\right)\right)\right) v\left(\left\{\omega_{i(1)}, \ldots, \omega_{i(q)}\right\}\right)
\end{aligned}
$$




$$
\begin{aligned}
& +\left(\sum_{i(k) \leq p \leq n-1}\left(x\left(\omega_{p}\right)-x\left(\omega_{p+1}\right)\right)\right) v(A)+x\left(\omega_{n}\right) v(A) \\
& =\sum_{1 \leq q \leq k-1}\left(x\left(\omega_{i(q)}\right)-x\left(\omega_{i(q+1)}\right)\right) v\left(\left\{\omega_{i(1)}, \ldots, \omega_{i(q)}\right\}\right)+x\left(\omega_{i(k)}\right) v(A) \\
& =\left.\left.\int_{A} x\right|_{A} d v\right|_{A} .
\end{aligned}
$$

The last formula is the Choquet integral on $A$. Thus, the proposition is shown since the Choquet integral on $A$ is $A$-conditionally comonotonic additive.

\section{Proof of Proposition 1}

Proof. Suppose that $\left.f\right|_{A}=\left.g\right|_{A}$. Then, by Proposition 2,

$$
\int_{\Omega} u(f) d v_{A}=\left.\int_{A} u\left(\left.f\right|_{A}\right) d v_{A}\right|_{A}=\left.\int_{A} u\left(\left.g\right|_{A}\right) d v_{A}\right|_{A}=\int_{\Omega} u(g) d v_{A} .
$$

Next, we show the converse. Note that $\left.1_{S}\right|_{A}=\left.1_{S \cap A}\right|_{A}$. Thus, by consequentialism, $1_{S} \sim_{A} 1_{S \cap A}$. So, $v_{A}(S)=\int_{\Omega} 1_{S} d v_{A}=\int_{\Omega} 1_{S \cap A} d v_{A}=v_{A}(S \cap A)$.

\section{Proof of Theorem 2}

Proof. (If part) We assume that there exist capacities $v$ and $v_{A}$ on $\mathcal{F}$ and a nonconstant affine real-valued function $u$ on $Y$ such that for all $f$ and $g$ in $L_{0}, f \succeq_{A}$ $g \Leftrightarrow \int_{\Omega} u(f(\omega)) d v_{A}(\omega) \geq \int_{\Omega} u(g(\omega)) d v_{A}(\omega)$, where it holds that $v_{A}(S)=v_{A}(S \cap A)$ for all $S \in \mathcal{F}$.

Clearly, Axiom 5 holds since $u$ is non-constant. Axiom 6 holds since $u$ does not depend on a certain event $A$. Axioms 1,3 , and 4 hold by Theorem 1 . Moreover, to show Axiom 7, note that $\left(y_{1},\{\omega\} ; y_{3}, \Omega \backslash\{\omega\}\right)=y_{1} 1_{\{\omega\}}+y_{3} 1_{\Omega \backslash\{\omega\}}$. Here, by $\omega \in A^{c}, 1_{\{\omega\}}$ and $1_{\Omega \backslash\{\omega\}}$ are $A$-conditionally comonotonic. Therefore, by Proposition $2, \int_{\Omega} u\left(y_{1},\{\omega\} ; y_{3}, \Omega \backslash\{\omega\}\right) d v_{A}=u\left(y_{1}\right) v_{A}(\{\omega\})+u\left(y_{3}\right) v_{A}(\Omega \backslash\{\omega\})$. Similarly, $\int_{\Omega} u\left(y_{2},\{\omega\} ; y_{3}, \Omega \backslash\{\omega\}\right) d v_{A}=u\left(y_{2}\right) v_{A}(\{\omega\})+u\left(y_{3}\right) v_{A}(\Omega \backslash\{\omega\})$. On the other hand, $v_{A}(\{\omega\})=v_{A}(\{\omega\} \cap A)=0$. Hence, Axiom 7 holds.

Finally, suppose that $f, g, h \in L_{0}$ are pairwise $A$-conditionally comonotonic and that $\int_{\Omega} u(f) d v_{A} \geq \int_{\Omega} u(g) d v_{A}$. Then,

$$
\begin{aligned}
& \int_{\Omega} u(\alpha f+(1-\alpha) h) d v_{A}-\int_{\Omega} u(\alpha g+(1-\alpha) h) d v_{A} \\
= & \alpha \int_{\Omega} u(f) d v_{A}+(1-\alpha) \int_{\Omega} u(h) d v_{A}-\alpha \int_{\Omega} u(g) d v_{A}-(1-\alpha) \int_{\Omega} u(h) d v_{A}
\end{aligned}
$$




$$
=\alpha\left(\int_{\Omega} u(f) d v_{A}-\int_{\Omega} u(g) d v_{A}\right) \geq 0,
$$

from the $A$-conditionally comonotonic additivity of $v_{A}$ shown in Proposition 2. Thus, Axiom 2 holds.

Now, by showing Lemmas 4, 5, and 11-14, we prove the only if part of Theorem 2. In the following, if the proofs for the unconditional preference $\succeq$ are the same as those for the conditional preferences $\succeq_{A}$, we omit them.

\section{Proof of Lemma 4}

Proof. If $f, g \in L_{0}$ are comonotonic, then $f$ and $g$ are $A$-conditionally comonotonic. Thus, Axiom 2 implies Comonotonic Independence Axiom. By Theorem 1, Comonotonic Independence Axiom together with Axioms 1, 3, 4, 5 implies that there exist a capacity $v_{A}$ on $\mathcal{F}$ and an affine real-valued function $u_{A}$ on $Y$ such that for all $f$ and $g$ in $L_{0}$,

$$
f \succeq_{A} g \Leftrightarrow \int_{\Omega} u_{A}(f(\omega)) d v_{A}(\omega) \geq \int_{\Omega} u_{A}(g(\omega)) d v_{A}(\omega)
$$

Moreover, by Axiom 6 , for $A$ and $\Omega$, we can show the existence of the common utility function $u$ such that it represents $\succeq$ and $\succeq_{A}$ on $L_{c}$ since $u$ is determined by the preference on $L_{c}$. Moreover, by Axiom 5, $u$ is non-constant.

\section{Proof of Lemma 5}

Proof. We prove this lemma by mimicking the arguments of Schmeidler $(1986,1989)$. Let $I(a)=\int_{\Omega} a(s) d v_{A}$. Let $a, b \in \mathbb{R}^{\Omega}$ be $A$-conditionally comonotonic. By Axiom 2 , for all pairwise $A$-conditionally comonotonic functions $a, b, c$, it holds that $I(a) \geq$ $I(b)$ implies $I(\alpha a+(1-\alpha) c) \geq I(\alpha b+(1-\alpha) c)$. First, let us prove the following claim: if $x, y \in \mathbb{R}^{\Omega}$ are $A$-conditionally comonotonic, then $I(\alpha x+(1-\alpha) y)=$ $\alpha I(x)+(1-\alpha) I(y)$ for all $\alpha \in[0,1]$.

Indeed, for any $\varepsilon>0,(I(x)+\varepsilon) 1_{\Omega}$ satisfies $I\left((I(x)+\varepsilon) 1_{\Omega}\right)>I(x)$ and $(I(y)+$ $\varepsilon) 1_{\Omega}$ satisfies $I\left((I(y)+\varepsilon) 1_{\Omega}\right)>I(y)$ since $I\left(\lambda 1_{\Omega}\right)=\lambda$. Hence,

$$
\begin{aligned}
& \alpha I(x)+(1-\alpha) I(y)+\varepsilon \\
= & I\left(\alpha(I(x)+\varepsilon) 1_{\Omega}+(1-\alpha)(I(y)+\varepsilon) 1_{\Omega}\right) \\
> & I\left(\alpha x+(1-\alpha)(I(y)+\varepsilon) 1_{\Omega}\right) \\
> & I(\alpha x+(1-\alpha) y) .
\end{aligned}
$$


The first inequality holds since $\alpha(I(x)+\varepsilon) 1_{\Omega}, x$, and $(1-\alpha)(I(y)+\varepsilon) 1_{\Omega}$ are pairwise $A$-conditionally comonotonic. The second inequality holds since $x, y$, and $(1-$ $\alpha)(I(y)+\varepsilon) 1_{\Omega}$ are pairwise $A$-conditionally comonotonic.

Since $\varepsilon$ is any positive number, we obtain that

$$
\alpha I(x)+(1-\alpha) I(y) \geq I(\alpha x+(1-\alpha) y) .
$$

Furthermore, the converse inequality can be shown by using a similar argument for $\varepsilon<0$. Therefore, it is proved that

$$
I(\alpha x+(1-\alpha) y)=\alpha I(x)+(1-\alpha) I(y) .
$$

Then, our claim is proved.

Next, let us use this claim twice. First, let $\alpha=1 / 2, x=2 a$, and $y=0$. Then, $I(a)=(1 / 2) I(2 a)$. Similarly, let $\alpha=1 / 2, x=0$, and $y=2 b$. Then, $I(b)=(1 / 2) I(2 b)$. Second, let $\alpha=1 / 2, x=2 a$, and $y=2 b$. Then,

$$
I(a+b)=\frac{1}{2} I(2 a)+\frac{1}{2} I(2 b)=I(a)+I(b) .
$$

Lemma 11. Let $v_{A}$ be the capacity in Lemma 4. Then, $v_{A}(S)=v_{A}(S \cap A)+v_{A}(S \cap$ $\left.A^{c}\right)$ for all $S \in \mathcal{F}$.

Proof. Note that $1_{S \cap A}$ and $1_{S \cap A^{c}}$ are $A$-conditionally comonotonic. By Lemma 5 , it holds that $\int_{\Omega} 1_{S \cap A}+1_{S \cap A^{c}} d v_{A}=\int_{\Omega} 1_{S \cap A} d v_{A}+\int_{\Omega} 1_{S \cap A^{c}} d v_{A}$. This equation implies that $v_{A}(S)=v_{A}(S \cap A)+v_{A}\left(S \cap A^{c}\right)$.

Lemma 12. Let $v_{A}$ be the capacity in Lemma 4. Then, $v_{A}(S)=\sum_{\omega \in S} v_{A}(\{\omega\})$ for every $S$ with $S \subseteq A^{c}$.

Proof. Let $T_{1} \subseteq A^{c}$ and $T_{2} \subseteq A^{c}$ satisfy $T_{1} \cap T_{2}=\emptyset$. Then, $1_{T_{1}}$ and $1_{T_{2}}$ are $A$ conditionally comonotonic. Hence, by Lemma 5, $\int\left(1_{T_{1}}+1_{T_{2}}\right) d v_{A}=\int 1_{T_{1}} d v_{A}+$ $\int 1_{T_{2}} d v_{A}$, which implies $v_{A}\left(T_{1} \cup T_{2}\right)=v_{A}\left(T_{1}\right)+v_{A}\left(T_{2}\right)$. Therefore, for every $S$ with $S \subseteq A^{c}$, it holds that $v_{A}(S)=\sum_{\omega \in S} v_{A}(\{\omega\})$ since $\Omega$ is finite.

Lemma 13. Let $v_{A}$ be the capacity in Lemma 4. Then, $v_{A}(S)=0$ for every $S \subseteq A^{c}$.

Proof. Take an arbitrary $y$ with $y^{*} \succ_{A} y \succ_{A} y_{*}$. Indeed, such $y^{*}, y$, and $y_{*}$ exist by A3, A5, and A6. And let $f=\left(y^{*},\{\omega\} ; y_{*}, \Omega \backslash\{\omega\}\right), g=\left(y,\{\omega\} ; y_{*}, \Omega \backslash\{\omega\}\right)$. By A7, it holds that $f \sim_{A} g$. Hence, $\int_{\Omega} u(f(\omega)) d v_{A}(\omega)=\int_{\Omega} u(g(\omega)) d v_{A}(\omega)$, which leads that $\left(u\left(y^{*}\right)-u\left(y_{*}\right)\right) v_{A}(\{\omega\})+u\left(y_{*}\right)=\left(u(y)-u\left(y_{*}\right)\right) v_{A}(\{\omega\})+u\left(y_{*}\right)$. Therefore, $v_{A}(\{\omega\})=0$ since $u\left(y^{*}\right)-u(y)>0$. This result together with Lemma 12 proves the claim. 
The following lemma is shown from Lemmas 11, 12, and 13, immediately.

Lemma 14. Let $v_{A}$ be the capacity in Lemma 4. Then, $v_{A}(S)=v_{A}(S \cap A)$ for all $S \in \mathcal{F}$.

\section{Proof of Lemma 6}

Proof. Let $f, g \in L_{0}$ and $A \in \mathcal{F} \backslash\{\Omega, \emptyset\}$ with $v\left(A^{c}\right) \neq 1$. For ease of notation, let $\Omega_{1}=\Omega^{U C}(f, g ; A)$. Note that for all $\omega \in \Omega_{1}$ and for all $\omega^{\prime} \in \Omega_{1}^{c}, f(\omega) \succeq f\left(\omega^{\prime}\right)$ and $g(\omega) \succeq g\left(\omega^{\prime}\right)$. Let $\left\langle\omega_{1}^{f}, \omega_{2}^{f}, \ldots, \omega_{n}^{f}\right\rangle$ be a permutation of $\Omega$ satisfying $u\left(f\left(\omega_{1}^{f}\right)\right) \geq$ $u\left(f\left(\omega_{2}^{f}\right)\right) \geq \cdots \geq u\left(f\left(\omega_{n}^{f}\right)\right)$. Similarly, let $\left\langle\omega_{1}^{g}, \omega_{2}^{g}, \ldots, \omega_{n}^{g}\right\rangle$ be a permutation of $\Omega$ satisfying $u\left(g\left(\omega_{1}^{g}\right)\right) \geq u\left(g\left(\omega_{2}^{g}\right)\right) \geq \cdots \geq u\left(g\left(\omega_{n}^{g}\right)\right)$. Let $\Omega_{1}=\left\{\omega_{1}^{f}, \ldots, \omega_{k}^{f}\right\}=$ $\left\{\omega_{1}^{g}, \ldots, \omega_{k}^{g}\right\}$. Then, by Axiom 8, it is possible to set $\omega_{i}^{f}=\omega_{i}^{g}$ for all $i \in\{1, \ldots, k\}$.

Note that

$$
\begin{aligned}
\int_{\Omega} u(f) d v_{A}^{D S} & =\sum_{i=1}^{n}\left(u\left(f\left(\omega_{i}^{f}\right)\right)-u\left(f\left(\omega_{i+1}^{f}\right)\right)\right) v_{A}^{D S}\left(\left\{\omega_{1}^{f}, \ldots, \omega_{i}^{f}\right\}\right) \\
\text { and } \int_{\Omega} u(g) d v_{A}^{D S} & =\sum_{i=1}^{n}\left(u\left(g\left(\omega_{i}^{g}\right)\right)-u\left(g\left(\omega_{i+1}^{g}\right)\right)\right) v_{A}^{D S}\left(\left\{\omega_{1}^{g}, \ldots, \omega_{i}^{g}\right\}\right),
\end{aligned}
$$

where $u\left(f\left(\omega_{n+1}^{f}\right)\right)=u\left(g\left(\omega_{n+1}^{g}\right)\right)=0$. Then, it follows that

$$
\begin{aligned}
& f \succeq_{A} g \Leftrightarrow \int_{\Omega} u(f) d v_{A}^{D S} \geq \int_{\Omega} u(g) d v_{A}^{D S} \\
\Leftrightarrow & \sum_{i=1}^{n}\left(u\left(f\left(\omega_{i}^{f}\right)\right)-u\left(f\left(\omega_{i+1}^{f}\right)\right)\right)\left(v\left(\left\{\omega_{1}^{f}, \ldots, \omega_{i}^{f}\right\} \cup A^{c}\right)-v\left(A^{c}\right)\right) \\
\geq & \sum_{i=1}^{n}\left(u\left(g\left(\omega_{i}^{g}\right)\right)-u\left(g\left(\omega_{i+1}^{g}\right)\right)\right)\left(v\left(\left\{\omega_{1}^{g}, \ldots, \omega_{i}^{g}\right\} \cup A^{c}\right)-v\left(A^{c}\right)\right) \\
\Leftrightarrow & \sum_{i=1}^{n}\left(u\left(f\left(\omega_{i}^{f}\right)\right)-u\left(f\left(\omega_{i+1}^{f}\right)\right)\right) v\left(\left\{\omega_{1}^{f}, \ldots, \omega_{i}^{f}\right\} \cup A^{c}\right)-u\left(f\left(\omega_{1}^{f}\right)\right) v\left(A^{c}\right) \\
& \geq \sum_{i=1}^{n}\left(u\left(g\left(\omega_{i}^{g}\right)\right)-u\left(g\left(\omega_{i+1}^{g}\right)\right)\right) v\left(\left\{\omega_{1}^{g}, \ldots, \omega_{i}^{g}\right\} \cup A^{c}\right)-u\left(g\left(\omega_{1}^{g}\right)\right) v\left(A^{c}\right),
\end{aligned}
$$

where the second equivalence holds by telescoping sums. Furthermore, by Axiom 8 , the summation from $i=1$ to $k-1$ of the first term on the left-hand side of the inequality is equal to that $0 n$ the right-hand side of the inequality and $u\left(f\left(\omega_{1}^{f}\right)\right) v\left(A^{c}\right)=u\left(g\left(\omega_{1}^{g}\right)\right) v\left(A^{c}\right)$. Moreover, by $A^{c} \subseteq\left\{\omega_{1}^{f}, \ldots, \omega_{k}^{f}\right\}=\left\{\omega_{1}^{g}, \ldots, \omega_{k}^{g}\right\}$, it follows that

$$
f \succeq_{A} g
$$




$$
\begin{aligned}
& \Leftrightarrow \sum_{i=k}^{n}\left(u\left(f\left(\omega_{i}^{f}\right)\right)-u\left(f\left(\omega_{i+1}^{f}\right)\right)\right) v\left(\left\{\omega_{1}^{f}, \ldots, \omega_{i}^{f}\right\}\right) \\
& \geq \sum_{i=k}^{n}\left(u\left(g\left(\omega_{i}^{g}\right)\right)-u\left(g\left(\omega_{i+1}^{g}\right)\right)\right) v\left(\left\{\omega_{1}^{g}, \ldots, \omega_{i}^{g}\right\}\right) .
\end{aligned}
$$

Note that by Axiom 8,

$$
\begin{aligned}
& \sum_{i=1}^{k-1}\left(u\left(f\left(\omega_{i}^{f}\right)\right)-u\left(f\left(\omega_{i+1}^{f}\right)\right)\right) v\left(\left\{\omega_{1}^{f}, \ldots, \omega_{i}^{f}\right\}\right) \\
= & \sum_{i=1}^{k-1}\left(u\left(g\left(\omega_{i}^{g}\right)\right)-u\left(g\left(\omega_{i+1}^{g}\right)\right)\right) v\left(\left\{\omega_{1}^{g}, \ldots, \omega_{i}^{g}\right\}\right) .
\end{aligned}
$$

By adding this to both sides of the above inequality, it follows that

$$
f \succeq_{A} g \Leftrightarrow \int_{\Omega} u(f) d v \geq \int_{\Omega} u(g) d v \Leftrightarrow f \succeq g .
$$




\section{References}

[1] Anscombe, F. and R. J. Aumann (1963): "A Definition of Subjective Probability," Annals of Mathematical Statistics 34, 199-205.

[2] Asano, T. and H. Kojima (2015): "An Axiomatization of Choquet Expected Utility with Cominimum Independence," Theory and Decision 78, 117-139.

[3] Dempster, A. P. (1967): "Upper and Lower Probabilities Induced by a Multivalued Mapping," Annals of Mathematical Statistics 38, 325-339.

[4] Dempster, A. P. (1968): "A Generalization of Bayesian Inference," Journal of Royal Statistical Society Series B 30, 205-232.

[5] Dominiak and J.-P. Lefort (2011): "Unambiguous Events and Dynamic Choquet Preferences," Economic Theory 46, 401-425.

[6] Dominiak, A., P. Duersch, and J.-P. Lefort (2012): "A Dynamic Ellsberg Urn Experiment," Games and Economic Behavior 75, 625-638.

[7] Eichberger, J., S. Grant, and D. Kelsey (2007): "Updating Choquet Beliefs," Journal of Mathematical Economics 43, 888-899.

[8] Ellsberg, D. (1961): "Risk, Ambiguity, and the Savage Axioms," Quarterly Journal of Economics 75, 643-669.

[9] Epstein, L. G. and M. Le Breton (1993): "Dynamically Consistent Beliefs Must be Bayesian," Journal of Economic Theory 61, 1-22.

[10] Epstein, L. G. and M. Schneider (2003): "Recursive Multiple-Priors," Journal of Economic Theory 113, 1-31.

[11] Fagin, R. and J. Y. Halpern (1991): "A New Approach to Updating Beliefs," in Bonissone, P. P., M. Henrion, L. N. Kanal, and J. F. Lemmer (eds.). Uncertainty in Artificial Intelligence 6, 347-374, Springer.

[12] Ghirardato, P. (2002): "Rivisiting Savage in a Conditional World," Economic Theory 20, 83-92.

[13] Ghirardato, P., F. Maccheroni, and M. Marinacci (2004): "Differentiating Ambiguity and Ambiguity Attitude," Journal of Economic Theory 118, 133-173.

[14] Ghirardato, P., F. Maccheroni, and M. Marinacci (2008): "Revealed Ambiguity and Its Consequences: Updating," in Abdellaboui, M. and J. D. Hey (eds.). Advances in Decision Making under Risk and Uncertainty, 3-18, Springer.

[15] Gilboa, I. (2009): Theory of Decision under Uncertainty, Cambridge University Press. 
[16] Gilboa, I. and D. Schmeidler (1989): "Maxmin Expected Utility with NonUnique Priors," Journal of Mathematical Economics 18, 141-153.

[17] Gilboa, I. and D. Schmeidler (1993): "Updating Ambiguous Beliefs," Journal of Economic Theory 59, 33-49.

[18] Hammond, P. (1988): "Consequentialist Foundations for Expected Utility," Theory and Decision 25, 25-78.

[19] Hammond, P. (1989): "Consistent Plans, Consequentialism, and Expected Utility," Econometrica 57, 1445-1449.

[20] Hanany, E. and P. Klibanoff (2007): "Updating Preferences with Multiple Priors," Theoretical Economics 2, 261-298.

[21] Hanany, E. and P. Klibanoff (2009): "Updating Ambiguity Averse Preferences," B. E. Journal of Theoretical Economics Vol. 9, Iss. 1 (Advances), Article 37.

[22] Horie, M. (2013): "Reexamination on Updating Choquet Beliefs," Journal of Mathematical Economics 49, 467-470.

[23] Jouini, E. and C. Napp (2004): "Conditional Comonotonicity," Decisions in Economics and Finance 27, 153-166.

[24] Kajii, A., H. Kojima, and T. Ui (2007): "Cominimum Additive Operators," Journal of Mathematical Economics 43, 218-230.

[25] Kreps, D. (1988): Notes on the Theory of Choice, Westview Press, Boulder.

[26] Machina, M. (1989): "Dynamic Consistency and Non-expected Utility Models of Choice under Uncertainty," Journal of Economic Literature 27, 1622-1668.

[27] Pires, C. P. (2002): "A Rule for Updating Ambiguous Beliefs," Theory and Decision 53, 137-152.

[28] Sarin, R. and P. Wakker (1998): "Dynamic Choice and Nonexpected Utility," Journal of Risk and Uncertainty 17, 87-119.

[29] Savage, L. J. (1954): The Foundation of Statistics. New York, Wiley. (Second edition in 1972, Dover.)

[30] Schmeidler, D. (1986): "Integral Representation without Additivity," Proceedings of the American Mathematical Society 97, 255-261.

[31] Schmeidler, D. (1989): "Subjective Probability and Expected Utility without Additivity," Econometrica 57, 571-587.

[32] Shafer, G. (1976): A Mathematical Theory of Evidence. Princeton University Press. 
[33] Siniscalchi, M. (2011): "Dynamic Choice under Ambiguity," Theoretical Economics 6, 379-421.

[34] Wang, T. (2003): "Conditional Preferences and Updating," Journal of Economic Theory 108, 286-321. 